\title{
Strikes and Slowdown in a Theory of Relational Contracts*
}

\author{
Robert Gary-Bobo ${ }^{\dagger}$ and Touria Jaaidane ${ }^{\ddagger}$
}

8 February 2011

\begin{abstract}
We propose a model of strikes in a relational (or self-enforcing) contracts framework. The employer has private information about firm profitability, proposes a wage and a bonus, and can outsource part of the production in each period. The union can either go on strike or reduce the workers's effort (i.e., decide a slowdown or workto-rule) as a response to a low wage or a low bonus. We construct perfect public equilibria in which strikes (or slowdown) appear randomly on the equilibrium path, during finite-duration spells triggered by the occurrence of a low-profitability state. Equilibria exhibit money-burning (i.e., conflict) and wage-compression as in the recent literature on relational contracts; they are first-best inefficient. We discuss some empirical implications of the model and applications to the public sector. An important advantage of our theory is that it allows for equilibrium regime changes, induced by changes in the environment: strikes may disappear and be replaced by other forms of conflict that are less easily observable. This has consequences for the empirical work on strikes.

JEL Class. No: C73, D2, J45, J5.

Keywords: Repeated Games, Imperfect Monitoring, Mechanism Design, Relational Contracts, Self-enforcing Contracts, Strikes, Slowdown, Industrial Relations, Public Employment.
\end{abstract}

* We thank Françoise Forges, Emeric Henry, Johannes Hörner, Francis Kramarz, Yossi Spiegel, and the audience of seminars held in Berlin, WZB and Paris, IEP Sciences Po, CREST-INSEE and Paris School of Economics for their help and comments. The usual caveat applies. The present paper is a deeply revised version of the manuscript circulated in 2006 and entitled "Strikes as the Tip of the Iceberg in a Theory of Firm-Union Cooperation"

$\dagger$ CREST-INSEE, 15 boulevard Gabriel Peri, 92245 Malakoff cedex, France and CEPR. Email: robert.gary-bobo@ensae.fr

$\ddagger$ Université de Lille 1, France. email: touria.jaaidane@univ-lille1.fr. 


\section{Introduction}

The theory of strikes is apparently outdated. True, in the past 10 or 15 years, the number of days lost to strikes has steadily decreased in the United States, and the UK record has been low in comparison with the European Union average $^{1}$; but strikes have not disappeared from continental Europe, their frequency has not decreased in India, and it seems that the number of wildcat strikes is quickly growing in China ${ }^{2}$. With the advent of the public debt crisis and the spreading of budgetary austerity, strikes could soon become a very significant problem in the Public Sector of many developed economies ${ }^{3}$. So, this topic may become fashionable again.

Labor disputes typically occur in ongoing, long-run relationships between an employer and the workers' union. Many strikes seem to be the result of a conflict situation that has been built up by past responses of the employer to changes in the environment ${ }^{4}$. It is very likely that strikes are only the "tip of the iceberg": that is, the visible part of the firm-union cooperation enforcement problem. Indeed, many internal conflicts never result in a strike that would be recorded by outside observers. Workers can use various forms of resistance and slowdown to obtain improvements of working conditions and pay. Resistance phenomena are less easily observable, but are likely to cause inefficiencies as severe as strikes. For instance, Krueger and Mas (2004) and Mas (2006) have shown that disappointed workers can cause a deterioration in the quality of produced goods and significant drops in some indirect measures of effort in general, even if labor strife is not recorded by outside observers.

Since the mid 1980's, the theory of strikes has been based mainly on models of bargaining under asymmetric information (see e.g., Kennan and Wilson (1989), (1993), Ausubel et al. (2001)). These models provide a powerful way of rationalizing labor disputes, but they cannot be easily extended to study dynamics, while the observed number of days lost to strikes is a time series exhibiting much randomness. In addition, there are good reasons to

\footnotetext{
${ }^{1}$ Hale (2008).

${ }^{2}$ Official statistics are not available. See however, e.g., The Economist (2010), and Albert Shanker Institute (2008).

${ }^{3}$ The Economist (2011).

${ }^{4}$ In the US, for instance, strikes are more likely the greater is uncompensated inflation over the previous contract period. See Vroman (1989).
} 
think that labor disputes can be understood in the framework of Relational (or Implicit) Contract Theory ${ }^{5}$.

The present paper proposes a simple way of integrating strikes in a theory of relational (or implicit) contracts between an employer and a workers' union, based on an infinitely repeated game with imperfect monitoring ${ }^{6}$. In this framework, conflict is the unavoidable consequence of informational asymmetries. But the various avatars of conflict, that is, strikes, slowdown, low morale, dismissals, resignations, etc., are not by themselves essential ingredients of the theory. Strikes, for instance, would have a nonzero incidence only under certain parameter configurations, while conflict is still present, but takes another form. This is important for the empirical work on labor disputes, because many aspects of conflict are not recorded in the data and therefore, the impact of factors causing strikes, as well as the real causal impact of labor strife in general, are hard to identify.

The proposed model explains the incidence of strikes, as well as the employer's outsourcing (or worker replacement) decisions. It also extends the theory to account for worker resistance phenomena, such as slowdown and "work-to-rule". In our model, strikes appear as random equilibrium phenomena. We show that high-effort and high-pay cooperative agreements between the union and the employer can be supported as Public Perfect Nash equilibria of a repeated game, if players are patient enough, but only at the cost of random reversions to inefficient sequences of actions, in which strikes and rigid wages, or slowdown and outsourcing may take place. In equilibrium, the union simply goes on strike when the proposed wage is too low and the real state of nature, being private information of the employer, is never revealed to the union. This type of union behavior is rational in a repeated interaction framework: it creates the necessary incentives for cooperation in good times. In the proposed model, strikes are not retrospective punishments inflicted on the employer when the real state of nature is disclosed, or partially revealed. In contrast, they are merely the response to a disappointing wage (or bonus) offer. But the main result is probably that this theory can explain changes of regime, in which strikes disappear. Our theory suggests that strikes vanish because they are replaced by other forms of conflict that are less easily

\footnotetext{
${ }^{5}$ On the latter theory, see e.g., Bull (1987), Malcomson and MacLeod (1989), Levin (2003).

${ }^{6}$ On this notion, see Fudenberg et al. (1994), Mailath and Samuelson (2006).
} 
observable. This happens when some structural parameters change, like, for instance, the cost of outsourcing.

The modern theory of strikes has first been developed with the help of Contract Theory, in static form (see e.g., Hayes (1984), and Card (1990)). Extensions of these ideas have been proposed by Cramton and Tracy (1992) and others, using games with incomplete information $^{7}$. These models typically describe a 3 -year contract-period. Bargaining over the wage rate takes place at the beginning of the time interval, and strikes are an inefficient delay, incurred before an agreement is signed. These models typically lack a dynamic element. It is intuitively clear that past contractual wages play a rôle in current contract negotiations, and that the current wage matters for the next contract. Changing circumstances in the input and output markets, unexpected inflation, and all sorts of business-cycle phenomena can randomly force players to renegotiate, at any moment ${ }^{8}$.

To the best of our knowledge, Robinson (1999) is the only contribution that proposed a model of labor disputes cast in an infinite-horizon, repeated-game, asymmetric-information framework, before the present paper. There are important differences in our respective ways of modelling the problem. Robinson (1999) adapts Green and Porter's (1984) oligopoly model, and models strikes as retrospective punishments: in each period $t$, the union receives a noisy signal of period $t-1$ 's true state of nature, and the union strikes for $T$ periods if it is sufficiently likely that the firm has lied about the state of nature in the past period. Our model, we believe, is more realistic: the true state of nature is never disclosed to the union; the union simply reacts to the employer's pay decisions: disappointing wages trigger reversion to an inefficient mode of play. Another important difference is the presence of the workers' effort variable. There is no effort (or slowdown) and no outsourcing (or partial lockout) in Robinson (1999), who makes the simpler assumption that the employer can replace the work force entirely, at a fixed cost $^{9}$.

\footnotetext{
${ }^{7}$ Again, see Ausubel et al. (2001)). The ability of bargaining models to reproduce a number of empirical facts about strikes is discussed in the papers of Kennan and Wilson (1989), and Card (1990).

${ }^{8}$ For a discussion of "within-contract strikes", see Harrison and Stewart (1994).

${ }^{9}$ There are a number of other questionable assumptions in Robinson's (1999) pionneering paper. He assumes that the employer is risk-averse and that the union is risk-neutral; he needs an upper bound on the player's discount factor to construct cooperative equilibria of his repeated game. We do not need these assumptions.
} 
Principal-Agent theory has been extended to repeated interaction settings to study worker moral hazard and efficiency wages (e.g., Malcomson and Spinnewyn (1988), McLeod and Malcomson (1989), (1998)), but this literature does not encompass strikes. The literature on subjective performance evaluation and self-enforcing contracts has then used the repeated game model to derive constraints on compensation systems (see e.g., Baker et al. (1994)). MacLeod (2003) models conflict in a rather abstract way as a form of money burning, but suggests interpretations in terms of possible employee behavior that can be the source of social costs, such as work-to-rule. Levin (2003) obtains related results in a repeated game context; in his analysis, conflict takes the form of worker resignation in termination contracts; he does not explore other forms of resource-consuming conflict. In these contexts, optimal contracts exhibit a property of "wage compression": we obtain the same kind of property here $^{10}$. It can be noted that the "stationary contracts" emphasized by Levin (2003) cannot be used in our model, because the rate at which value can be transfered from the Principal (Employer) to the Agent (Union) depends on the privately observed state of nature. Our model shows that wage compression and money burning can be generated as equilibrium phenomena in simple repeated mechanism design settings with private information, but in which private monitoring plays no role.

In the following, Section 2 sketches a case study of the municipal dustmen strikes, and discusses some empirical evidence, for illustrative purposes; Section 3 is devoted to a description of the model. Section 4 presents the construction of equilibria in the repeated game model and their properties are discussed in Section 5. A number of proofs are gathered in the Appendix.

\footnotetext{
${ }^{10}$ Since the first version of the present paper was written (Gary-Bobo and Jaaidane (2006)), some new results have appeared in the literature on subjective performance evaluation. Fuchs (2007) extends previous contributions in a repeated game setting with private monitoring: he confirms that termination contracts and money burning are second-best optimal. Rui Zhao (2009) explicitly considers low effort spells as a substitute for termination contracts and interprets low effort as low worker morale.
} 


\section{Dustmen in the City of Paris: Sketch of a Case Study}

We begin by briefly discussing labor disputes between municipal dustmen (i.e., sanitation workers) and the city of Paris, France. This is the type of implicit-contract, long-run relationship between a firm and a union that we try to model below. Some original data on the strikes and wages of these workers, recently collected by the authors, will provide an empirical illustration and a motivation for the theoretical analysis.

The dustmen of Paris enjoy a civil servant status which guarantees life-time employment to all workers: once hired, dustmen cannot be fired, except for very serious causes. Due to strict seniority rules and because firing is essentially impossible, when it comes to personnel motivation, the city of Paris is placed in an uncomfortable position. A single powerful trade union, the CGT, negotiates with the town hall ${ }^{11}$. The town hall cannot lock dustmen out, or use replacement workers, but they can rely on outsourcing to private sector sanitation companies, using public procurement contracts. The only problem is that this process takes time, to auction the contract and to build new garbage trucks. It can thus be very costly to outsource a large number of tons of garbage in a short period of time. The army has been used to clean up the streets during some famous strikes of the past.

Figure 1 plots the dustmen's number of strike days, per year, from 1968 to 2004 . It seems that the series is non-stationary, with a regime switch in the early eighties. The recurrent strikes of the late 1960's and early 1970's had resulted in important pay raises, and many new recruits. A new Mayor, Mr Jacques Chirac (a prominent politician, who was later to become the President of the Republic), was elected in 1977. Mayor Chirac's election was greeted with very tough strikes in 1978 and 1979. Until 1977, the city of Paris had never had recourse to the private sector: Mayor Chirac and his team crossed the Rubicon, and started partial privatization of garbage collection in the early eighties. Figure 1 clearly shows the reduction in strike incidence, starting from this period. Strikes have almost disappeared in the nineties, during Jacques Chirac's second and third terms (with the exception of 1990). He stayed in office from 1977 to 1995 and was replaced by a deputy mayor from the same party

\footnotetext{
${ }^{11}$ CGT stands for Confédération générale du travail. Note that, in France, union certification does not lead to union monopoly or exclusive representation in bargaining units, but the CGT is the dominant union in the case of dustmen.
} 
until 2001. Thus, the outsourcing-privatization policy has lasted long enough to become a well-established, credible mechanism. Its strategic effect on strikes seems obvious. A glance at Figure 2 shows the year to year change in the total number of dustmen, (as voted by the Town Hall, and published in its official record). From 1978 to 2000, with the exception of 1982 and 1983, the total number of dustmen remained constant and then decreased. In the year 2001, a new mayor from the Socialist Party, with a public-sector friendly approach, took office. In any case, new recruits had to be hired to compensate for the implementation of the 35-hours week law, which had just been passed by the Socialist government. A resurgence of strikes seems to have been the result of this policy.

But there is an unobserved downside to Mayor Chirac's (apparently successful) policy, which is that, since incentives are weak in the civil service, the privatization and low-pay policy have created the conditions of chronic low effort, and progressively demoralized the workers. We have many reasons to believe that the rate of absenteeism, which is abnormally high among municipal dustmen nowadays, has in fact increased over time. Slowdown or work-to-rule spells became more frequent and are not recorded as strikes ${ }^{12}$. So, the drop in strike incidence that we observe in Fig. 1 after 1983 could be due to a change of regime (i.e., a change in the implicit contract). We suggest below that these facts can be explained by a change in the form of conflict involved in (or used to support) the long-run equilibrium. In other words, the underpinning of the implicit contract between the town hall and the union has shifted from observable strikes to unobservable slowdown problems. In Appendix A, we provide further details on the Paris dustmen case, and show that drops in the discounted value of careers seem to cause strikes. The data on this case doesn't contradict the view that the discounted value of future wages and working conditions is the driving force behind the observed history of disputes.

To sum up, these empirical facts, taken together, suggest that they have been generated by a long-run relationship between the town hall and the dustmen's union. It seems that a finite-horizon, single-contract bargaining game with incomplete information cannot reproduce the facts as accurately as a model of repeated interaction, in which pay raises,

\footnotetext{
${ }^{12}$ Our inquiry has revealed that, to an extent which is difficult to measure or establish objectively, the quality of applicants for vacant public dustmen positions has also decreased in the recent years. For details on Paris dustmen, see Jaaidane and Gary-Bobo (2008).
} 
strikes, slowdown, outsourcing and the business cycle combine to produce a seemingly random sequence of conflicts.

\section{A Repeated Game Model of Slowdown and Strikes}

We consider an infinitely repeated game with 2 players: the employer and the union ${ }^{13}$.

\subsection{Basic Assumptions}

During each period, $t$, the Union and the Employer play a stage game $G$ : the Union chooses a level of effort $e_{t}$ in the set $\{\underline{e}, \bar{e}\}$ and a "length," or probability of strike $s_{t}$ in the interval $[0,1]$. We assume that the Union has enough command of its members to implement the effort and strike policy. The effort variable is also the employee's disutility of effort ${ }^{14}$.

Effort determines the total number of hours, $h$, needed to complete the task. High effort $\bar{e}$ is associated with a small number of hours $\underline{h}$, while low effort $\underline{e}$ corresponds to a large number of hours $\bar{h}>\underline{h}$. In contrast, strikes are complete work stoppages during which a fraction $s_{t}$ of output is lost. Our most important assumption is the following:

Assumption 1. $\bar{e} \underline{h}<\underline{e} \bar{h}$.

Assumption 1 says that the social cost of low effort is higher than that of high effort, as will become clear below. The employer chooses a real wage rate $w_{t}$ in each period $t$ and simultaneously, chooses the extent of outsourcing $x_{t}$ in the interval $[0,1]$. To be more precise, a fraction of the task $x_{t}$, in the interval $[0,1]$, can be outsourced. Accordingly, the fraction of the task fulfilled by the employees is $1-x_{t}$. We also assume that outside options and (or) rules concerning the workers impose that the real wage rate $w_{t}$ cannot fall below a minimum $\underline{w}$, which is assumed constant for simplicity. To focus on the interesting cases, we assume the following.

\footnotetext{
${ }^{13}$ To fix ideas, the employer can be viewed as a public entity, the "town hall", and the union is that of dustmen and sanitation workers. The employer has a given task to accomplish: a given, constant number of tons of waste, say, has to be collected during each time period.

${ }^{14}$ Intuitively, in the absence of incentives, low effort $\underline{e}$ is the natural behavior of workers, but, following a good agreement, the worker's morale can be raised by the Union's officials, and high effort $\bar{e}>\underline{e}$ can be implemented.
} 
Assumption 2. $\quad 0<\underline{e}<\bar{e} \leq \underline{w}$.

The cost of outsourcing is,

$$
C(x)=\underline{w h} x+\beta \frac{x^{2}}{2},
$$

where $\beta>0$. This formulation rests on the idea that subcontracting companies are efficient, since they pay the minimum wage $\underline{w}$ and obtain a high level of effort from their workers, i.e., $h=\underline{h}$, but that there are additional outsourcing costs, represented by the quadratic term. This latter term captures the fact that outsourcing is a source of specific organizational costs. It is likely that subcontractors are in a better bargaining position to extract surplus from the employer when the extent of outsourcing is large. This quadratic term may also represent the "political costs" of replacing civil servants with private sector workers.

Now, the employer faces changing circumstances, a changing state of the world, that we model as an i.i.d random cost parameter $\theta_{t}$ - interpreted as a "cost of public funds" in the case of a public employer. This cost parameter is observed by the employer but not by the union. For simplicity, $\theta_{t}$ takes only two values: $\underline{\theta}$, the "good state", with probability $\pi$, and a high value $\bar{\theta}>\underline{\theta}$, the "bad state", with probability $1-\pi$. The cost of public funds summarizes a number of random phenomena: business cycle fluctuations, price changes in product and input markets ${ }^{15}$, etc. In the stage game $G$, at time $t$, the union chooses $\left(e_{t}, s_{t}\right)$, and the employer simultaneously chooses a state-dependent action, a mapping $\theta \rightarrow\left(x_{\theta t}, w_{\theta t}\right)$.

We can now specify the players' payoffs in the stage game. We drop the time index $t$ to simplify notation. The union's payoff in $G$ and in state $\theta$ is defined as follows,

$$
u_{\theta}=(1-s)\left(1-x_{\theta}\right)\left(w_{\theta}-e\right) h
$$

Note that $u_{\theta}$ depends on the state only through the employer's state-contingent actions $\left(w_{\theta}, x_{\theta}\right)$. The interpretation of this specification is easy. The worker's surplus per hour $\left(w_{\theta}-e\right)$ is multiplied by the required number of hours $h$ and by the fraction of the task (the

\footnotetext{
${ }^{15}$ In the case of a public employer, cycles cause changes in tax revenues, changes in the interest rate on public debt, but there are also unexpected costs faced by the town hall, and changes in the priorities or in the political agenda of the ruling mayor's team, which change the tradeoffs that they face, in view of reelection. All these factors are subsumed in parameter $\theta_{t}$, which modifies the employer's resistance to the union's demands.
} 
number of tons of waste) carried out by the union's workers in state $\theta$, that is $\left(1-x_{\theta}\right)$. We assume that the workers receive a zero wage (and exert effort zero) during strikes, so that the union's total surplus is multiplied by $(1-s)$, the proportion of non-strike days.

The employer maximizes the (social) value of the task, denoted $v_{0}$, minus internal production costs, minus the cost of outsourced services. The fraction of the task performed by the union and the external providers taken together is $(1-s)(1-x)+x=1-s+x s$. When the workers are on strike, the external provider produces a fraction $s x$ of the task. The remaining part of the task, $1-x-(1-s)(1-x)=s(1-x)$, is not fulfilled ${ }^{16}$. The total cost of production is defined as follows.

$$
\theta\left[w_{\theta} h(1-s)\left(1-x_{\theta}\right)+C\left(x_{\theta}\right)\right]
$$

where $C$ is the cost of outsourcing defined above. The employer's utility in state $\theta$ is denoted $v_{\theta}$, and defined as follows:

$$
v_{\theta}=v_{0}\left(1-s+s x_{\theta}\right)-\theta\left[w_{\theta} h(1-s)\left(1-x_{\theta}\right)+C\left(x_{\theta}\right)\right]
$$

The social surplus is $R_{\theta}=u_{\theta}+v_{\theta}$, the sum of the players' stage game utilities. We have,

$$
R_{\theta}=v_{0}\left(1-s+s x_{\theta}\right)+\left((1-\theta) w_{\theta}-e\right)(1-s)\left(1-x_{\theta}\right) h-\theta C\left(x_{\theta}\right) .
$$

To simplify the presentation we propose the following normalization of parameters.

Assumption 3. $1=\underline{w}=\underline{h}=\bar{e}=\underline{\theta}<\bar{\theta}$.

As a consequence, under Assumption 2, we have $1=\bar{e}>\underline{e}$. These assumptions are harmless, except $1=\underline{\theta}$, because this ensures that one unit of money from the employer is worth exactly one unit for the union in the "good state" $\underline{\theta}$. It follows that surplus is perfectly transferable in the good state, while in the bad state, $\bar{\theta}>1$ acts as a "cost of public funds"17.

\footnotetext{
${ }^{16}$ In the waste collection example, $s(1-x)$ is the fraction of the garbage that is collected neither by the public, nor by the private sector dustmen, and stays in the streets (forcing citizens, or the army, to do the job).

${ }^{17}$ Since $R_{\theta}$ doesn't depend on the wage $w_{\theta}$ when $\theta=1$, the essential role of this latter assumption is to simplify the analysis. Note that a worker exerting high effort and paid the minimum wage has a zero rent, since $\underline{w}-\bar{e}=0$.
} 
Let now $\delta$ be the players' common discount factor. In the infinitely repeated game, denoted $G(\delta)$, the union's and employer's expected payoffs are respectively defined as follows:

$$
U=(1-\delta) \sum_{t=0}^{\infty} \delta^{t} E\left(u_{t \theta}\right), \quad V=(1-\delta) \sum_{t=0}^{\infty} \delta^{t} E\left(v_{\theta t}\right),
$$

where the expectation $E($.$) is taken with respect to state \theta$ 's distribution and $0<\delta<1$.

\subsection{Noncooperative Nash equilibrium of the stage game}

To solve the stage game $G$, we first analyze the employer's best reply to a given $(e, s)$. The employer's strategy is to offer a state-dependent wage and outsourcing level pair $\left(w_{\theta}, x_{\theta}\right)$. We therefore solve for a standard Bayesian-Nash equilibrium of $G$.

\subsubsection{The employer's best reply}

The employer's best reply is a pair of functions, $\left(w_{\theta}^{*}(e, s), x_{\theta}^{*}(e, s)\right)$. Given that $v_{\theta}$ is a nonincreasing function of $w_{\theta}$, the choice $w_{\theta}^{*}=\underline{w}$ is always a best response, and is the unique best response if $x^{*}<1$ and $s^{*}<1$. The best reply $x_{\theta}^{*}(e, s)$ is the value of $x_{\theta}$ which maximizes $v_{\theta}$. Using Assumption 3 and the result $w_{\theta}^{*}=\underline{w}=1$, the first-order condition for an interior maximum can be written,

$$
v_{0} s+\theta h(1-s)-\theta-\theta \beta x_{\theta}^{*}(s, e)=0,
$$

from which we immediately derive,

$$
x_{\theta}^{*}(s, e)=\frac{1}{\theta \beta}\left[v_{0} s+\theta h(1-s)-\theta\right]^{+},
$$

where for any $z$, we denote $[z]^{+}=\max \{0, z\}$. Remark that $x_{\theta}^{*}$ is a function of $e$ because $h$ is a function of $e$. In the following, we assume that $\beta$ is large enough so as to obtain ${ }^{18} x_{\theta}^{*}<1$. Since $v_{\theta}$ is a concave function of $x_{\theta}$, the above necessary solution is also sufficient.

\subsubsection{The union's equilibrium strategy}

The Union chooses the pair $\left(s^{*}, e^{*}\right)$ so as to maximize the expected utility $E\left[u_{\theta}\right]$, that is,

$$
(\underline{w}-e) h(1-s)\left(1-E x_{\theta}^{*}\right) .
$$

\footnotetext{
${ }^{18}$ For instance, we can take $\beta>v_{0}+\overline{\theta h}-1$ to guarantee this result.
} 
Given that we have $0 \leq E x_{\theta}^{*}<1$, a best response maximizes $(\underline{w}-e) h(1-s)$, taking $E x_{\theta}^{*}$ as given. It is straightforward that this implies $s^{*}=0$ and $e^{*}=\underline{e}$ if and only if $(\underline{w}-\underline{e}) \bar{h}>(\underline{w}-\bar{e}) \underline{h}=0$. But the latter condition is true under Assumptions 1-3. As a consequence, we can state the following result.

Result 1. The Nash equilibrium of the stage game $G$, denoted $\left(e^{*}, s^{*}, w_{\theta}^{*}, x_{\theta}^{*}\right)$, is unique and we have,

$$
e^{*}=\underline{e}, \quad s^{*}=0, \quad w_{\theta}^{*}=\underline{w}, \quad x_{1}^{*}=x_{\bar{\theta}}^{*}=(\bar{h}-1) / \beta .
$$

In other words, the one-shot Nash equilibrium is characterized by a positive amount of outsourcing, low effort, low pay and no strikes ${ }^{19}$. The expected utilities in this equilibrium are denoted $\left(u^{*}, v^{*}\right)$. We have,

$$
\begin{aligned}
& u^{*}=E u_{\theta}^{*}=(1-\underline{e}) \bar{h}\left(1-x_{1}^{*}\right), \\
& v^{*}=E v_{\theta}^{*}=v_{0}-E(\theta)\left[\bar{h}\left(1-x_{1}^{*}\right)+C\left(x_{1}^{*}\right)\right] .
\end{aligned}
$$

For future reference, define

$$
\mu=E(\theta)=(1-\pi) \bar{\theta}+\pi
$$

\subsection{Cooperative solutions in the stage game $\mathrm{G}$}

By definition, a cooperative solution of $G$ maximizes the expected surplus $E R_{\theta}=E\left(u_{\theta}+v_{\theta}\right)$ with respect to $\left(e, s, w_{\theta}, x_{\theta}\right)$, subject to the constraints: $e \in\{\underline{e}, \bar{e}\}, 0 \leq s \leq 1,1 \leq w_{\theta} \leq v_{0}$ and $0 \leq x_{\theta} \leq 1$. A cooperative solution maximizes the expected value of $R$, taking into account the fact that the employer knows the state of nature while choosing $w$ and $x$, and can be described as follows: the union commits to a policy $(e, s)$, and the employer, in exchange, chooses $\left(w_{\theta}, x_{\theta}\right)$ once the cost of funds $\theta$ is known. Let $\left(e^{c}, s^{c}, w_{\theta}^{c}, x_{\theta}^{c}\right)$ denote the cooperative solution.

We first maximize $R_{\theta}$ with respect to $\left(w_{\theta}, x_{\theta}\right)$ for each state $\theta$. First, if $\theta=\underline{\theta}=1$, then, $R_{\theta}$ doesn't depend on $w_{1}$ and the solution, denoted $w_{1}^{c}$, is indeterminate. Intuitively, there

\footnotetext{
${ }^{19}$ If $\bar{h}$ is large, and $\beta$ is small, the Nash equilibrium is a near collapse of the firm: cooperation is almost a necessary condition of the firm's existence.
} 
will be an interval of possible cooperative wages in the good state. Define the cooperative bonus

$$
b=w_{1}^{c}-\underline{w} .
$$

This bonus is a socially costless transfer between the employer and the union in the good state.

Second, if $\theta=\bar{\theta}>1$, then, $R_{\theta}$ is a nonincreasing function of $w$, strictly decreasing if $x$ and $s<1$. Efficiency then requires $w_{\bar{\theta}}^{c}=\underline{w}$. In the bad state, a pay raise is too costly and the union should accept austerity, in the name of efficiency. This will of course be a source of conflict.

We now search for the best cooperative amount of outsourcing $x_{\theta}^{c}(s, e)$. Note that $R_{\theta}$ is concave with respect to $x_{\theta}$, so that necessary conditions for optimality are also sufficient. The first-order condition for surplus maximization in state $\theta$ can be written,

$$
v_{0} s+\left(e-(1-\theta) w_{\theta}^{c}\right) h(1-s)-\theta-\theta \beta x_{\theta}^{c}(s, e)=0
$$

which immediately yields,

$$
x_{\theta}^{c}(e, s)=\frac{1}{\theta \beta}\left[v_{0} s+\left(e-(1-\theta) w_{\theta}^{c}\right) h(1-s)-\theta\right]^{+} .
$$

Again, we assume that $\beta$ is large enough to ensure that $x_{\theta}^{c}(s, e) \leq 1$.

To maximize expected surplus $E R_{\theta}$ with respect to $s$, given $\left(w_{\theta}^{c}, x_{\theta}^{c}\right)$, we compute the derivative,

$$
\frac{d E R_{\theta}}{d s}=E\left(\frac{\partial R_{\theta}}{\partial s}\right)+E\left(\frac{\partial R_{\theta}}{\partial x_{\theta}} \frac{\partial x_{\theta}^{c}}{\partial s}\right)=E\left(\frac{\partial R_{\theta}}{\partial s}\right) .
$$

The right-hand side equality is due to the envelope theorem: if $x_{\theta}^{c} \equiv 0$ on some open neighborhood, then $\partial x_{\theta}^{c} / \partial s=0$, and if $x_{\theta}^{c}>0$, then, by definition, $\partial R_{\theta} / \partial x_{\theta}=0$. Thus, using the definition of $R_{\theta}$, we have,

$$
\frac{d E R_{\theta}}{d s}=E\left(\left(1-x_{\theta}^{c}\right)\left[(\theta-1) h w_{\theta}^{c}+e h-v_{0}\right]\right) .
$$

Given that $x_{\theta}^{c}<1$, the above derivative is negative if $v_{0}$ is large enough, that is, if there is enough surplus. We therefore assume,

Assumption 4. $\quad v_{0}>(\bar{\theta}-1) \bar{h}+\underline{e} \bar{h}$. 
Under Assumption 4, we immediately obtain $s^{c}=0$. It is now possible to prove the following result.

\section{Result 2.}

Under Assumptions 1-4, there exists a value $\beta_{0}>0$, such that if $\beta>\beta_{0}$, the cooperative solutions of $G$ are characterized by the following properties: $e^{c}=\bar{e}, s^{c}=0, x_{1}^{c}=x_{\bar{\theta}}^{c}=0$, $w_{\bar{\theta}}^{c}=\underline{w}=1$ and $w_{1}^{c}$ is indeterminate.

For proof, see Appendix B.

We conclude that efficient cooperative agreements involve no strikes, high effort, no outsourcing, a minimal wage in the bad state, and a high compensation in the good state. Intuitively, the wage $w_{1}^{c}$ must be high enough in "good times," when $\theta=1$, to compensate workers for the effort, and for austerity in "bad times".

\section{Construction of Equilibria in the Repeated Game}

Our model is a game of repeated adverse selection. In these games, the moves of players are public information, but moves are taken after players learn some private information. An instance of this class of games is Athey and Bagwell's (2001) model of repeated pricecompetition oligopoly, in which firm prices are public, but firm costs are subject to privately observed i.i.d. shocks. In these games, observed moves are public signals of the player's actions, and the actions map the set of private types into the set of possible moves: these mappings are not observed. In our model, the employer's moves $(w, x)$ are observed by the union, and are therefore public information, but $\theta$ is a private i.i.d. shock which is never disclosed. An employer's pure action at time $t$ is a mapping of the set of types $\{\underline{\theta}, \bar{\theta}\}$ into the set of possible moves $\left[\underline{w}, v_{0}\right] \times[0,1]$. Employer actions are not observed by the union. Based on this formal definition of an action, games of repeated adverse selection can be reformulated as repeated games with imperfect public monitoring ${ }^{20}$. Thus, a number of

\footnotetext{
${ }^{20}$ Fudenberg, Maskin and Levine (1994) have shown that a repeated game in which (i), the players' types are private information, (ii) new types are drawn independently at each stage, and (iii), the players' actions are publicly observed, can formally be reformulated as a repeated game with imperfect public monitoring,
} 
general results and techniques can be applied to them. Since $\theta$ is never disclosed, $G(\delta)$ has no proper subgames, and it follows that an appropriate equilibrum concept is the Perfect Public Nash Equilibrium (hereafter PPE), in which players play public strategies, that is, by definition, strategies which depend only on publicly observable histories of play, but do not depend on past unobservable types and actions of the player ${ }^{21}$. A PPE is defined as a profile of public strategies that, after any public history, specifies a Nash equilibrium for the repeated game. On these questions, see Abreu et al. (1990), Fudenberg et al. (1994), Mailath and Samuelson (2006).

In the following, we will first show that the repeated play of certain actions $\widehat{\sigma}$, involving strikes, is a PPE of the repeated game $G(\delta)$, using trigger strategies. We then show that cooperative play $\sigma^{c}$ can be supported as part of a PPE, when players are sufficiently patient, but at the cost of random reversions to $\widehat{\sigma}$, involving strikes and rigid wages. Cooperative play can also be supported by random reversions to the static Nash equilibrium $\sigma^{*}$ during a finite number of periods, in which strikes do not occur, but in which effort is low and outsourcing takes place. So, slowdown and (or) strikes, and partial replacement of workers, are ways of supporting cooperation in a repeated-interaction, asymmetric-information context.

\subsection{Construction of an equilibrium with rigid wages and strikes}

We start our study of the repeated game by showing that there exists equilibria with rigid wages and in which strikes occur during a positive fraction of each period $t$. Let $\widehat{\sigma}=$ $(\widehat{e}, \widehat{s}, \widehat{w}, \widehat{x})$ be a list of actions of $G$ such that $\widehat{s} \geq 0 ; \widehat{e}=\bar{e}=1 ; \widehat{x}_{\theta}=0$ for all $\theta ; \widehat{w}_{\theta}=\widehat{w}$ for all $\theta$ and $\widehat{w}>1$. The stage game payoffs of $\widehat{\sigma}$ are easy to compute, we have, $\widehat{u}=(1-\widehat{s})(\widehat{w}-\bar{e})$, $\widehat{v}_{\theta}=(1-\widehat{s})\left(v_{0}-\theta \widehat{w}\right)$ and $\widehat{v}=E \widehat{v}_{\theta}=(1-\widehat{s})\left(v_{0}-\mu \widehat{w}\right)$. From this we immediately derive the if a player's payoff does not directly depend on the other players' types. In other words, a repeated adverse selection problem under private values can formally be rewritten in the form of a repeated game with imperfect monitoring, i.e., a repeated multi-person moral hazard model.

${ }^{21}$ With public strategies, the employer's choices at time $t$ can depend on the current, privately observed state $\theta_{t}$, through the action chosen at time $t$, but may not depend on past actions, and may depend on past states of nature only through past moves. Given our assumptions, the union can only play public strategies and it is easy to show that the employer has a best reply which is a public strategy too. In this context, there is no loss of generality in restricting the analysis to public strategies and public equilibria (again see Mailath and Samuelson (2006)). 
long-run payoffs $(\widehat{U}, \widehat{V})$, as follows:

$$
\begin{aligned}
\widehat{U} & =(1-\delta) \sum_{t=0}^{\infty} \delta^{t} \widehat{u}=\widehat{u}=(1-\widehat{s})(\widehat{w}-1), \\
\widehat{V} & =(1-\delta) \sum_{t=0}^{\infty} \delta^{t} \widehat{v}=\widehat{v}=(1-\widehat{s})\left(v_{0}-\mu \widehat{w}\right) .
\end{aligned}
$$

The associated total surplus is $\widehat{R}=\widehat{U}+\widehat{V}=(1-\widehat{s})\left(v_{0}-(\mu-1) \widehat{w}-1\right)$.

Perpetual repetition of the stage-game Nash equilibrium $\sigma^{*}=\left(e^{*}, s^{*}, w^{*}, x^{*}\right)$ is obviously a PPE of $G(\delta)$. The payoffs of this equilibrium, denoted $\left(U^{*}, V^{*}\right)$ are simply $U^{*}=u^{*}$ and $V^{*}=v^{*}$, defined above by (8). We also define the total surplus $R^{*}=U^{*}+V^{*}=$ $v_{0}-(\mu-1+\underline{e}) \bar{h}\left(1-x_{1}^{*}\right)-\mu C\left(x_{1}^{*}\right)$. We now show that there exists admissible $(\widehat{s}, \widehat{w})$ such that $\widehat{R}>R^{*}$. To do this, we set $\widehat{s}=0$ and choose the value of $\widehat{w}$ so as to ensure $\widehat{u}>u^{*}$ and $\widehat{v}>v^{*}$. This imposes,

$$
1+(1-\underline{e}) \bar{h}\left(1-x_{1}^{*}\right)<\widehat{w}<1+\bar{h}\left(1-x_{1}^{*}\right)+C\left(x_{1}^{*}\right)
$$

Computing the difference between the upper and the lower bounds, we easily show that this open interval for $\widehat{w}$ is nonempty if and only if,

$$
\left(1-x_{1}^{*}\right)(\underline{e} \bar{h}-1)+\beta \frac{\left(x_{1}^{*}\right)^{2}}{2}>0
$$

which is always true under Assumption 1. Now, given a feasible $\widehat{w}$, by continuity, we can always find a value $\widehat{s}>0$ small enough to preserve $\widehat{u}>u^{*}$ and $\widehat{v}>v^{*}$. The minimal value of $\widehat{w}$, denoted $\widehat{w}^{*}$, is given by the following expression,

$$
\widehat{w}^{*}=1+\frac{(1-\underline{e}) \bar{h}\left(1-x_{1}^{*}\right)}{(1-\widehat{s})}>1 .
$$

We conclude that there exist values $(\widehat{s}, \widehat{w})>>(0,1)$ such that, at the same time, $\widehat{U}>U^{*}$ and $\widehat{V}>V^{*}$.

We must now show that the perpetual repetition of $\widehat{\sigma}=(\widehat{e}, \widehat{s}, \widehat{w}, \widehat{x})$ is a trigger strategy equilibrium of $G(\delta)$ if $\delta$ is high enough. This is fairly standard. Consider the following strategies: both players initially play $\widehat{\sigma}$ and any deviation is punished by a permanent reversion to $\sigma^{*}$. The equilibrium condition for the union is $\widehat{U} \geq(1-\delta) u^{\prime}+\delta U^{*}$, where $u^{\prime}$ is the expected payoff induced by the union's best one-shot deviation in the stage game, when 
the employer plays $(\widehat{w}, \widehat{x})$. If $\widehat{U}>U^{*}$, there exists a $\delta_{0}<1$ such that the condition holds for $\delta>\delta_{0}$. The reasoning is the same for the employer. There are no inference problems in this equilibrium because all deviations are perfectly detected by both players. We conclude that when players are sufficiently patient and $\beta$ is sufficiently large, the repetition of $\widehat{\sigma}$ is an equilibrium.

Result 3. Under Assumptions 1-4, there exists a $\delta_{0}<1$ and $a \beta_{0}>0$, such that, if $1>\delta>\delta_{0}$ and $\beta>\beta_{0}$, the perpetual repetition of $\widehat{\sigma}$ is a PPE path of $G(\delta)$.

This equilibrium entails a permanent, positive duration of strikes, no outsourcing, high effort and constant wages above the minimum. It is potentially very inefficient, because efficient arrangements require no strikes and a state-contingent, flexible-wage policy. In this type of equilibrium, strikes seem to be the cause of above-the-minimum wages. As shown by the formula for $\widehat{w}^{*}$ above, there is a trade-off between equilibrium wages and equilibrium strikes. In a cross-section of firms, during phases in which $\widehat{\sigma}$ is played, one could observe an increasing relationship between strikes and wages, but the observed effect of strikes on wages is not "causal". We now turn to a family of equilibria that are much more efficient than the rudimentary $\widehat{\sigma}$.

\subsection{A set of cooperative equilibria in which strikes are random events}

We now construct an equilibrium involving the cooperative mode of play $\sigma^{c}=\left(e^{c}, s^{c}, w^{c}, x^{c}\right)$ defined above. We need a precise definition of our equilibrium candidate's strategies.

\section{Definition (Equilibrium Candidate):}

(i) In the initial period, $t=0$, the union cooperates and plays $\left(e^{c}, s^{c}\right)=(1,0)$. In state $\theta=1$, the employer plays $\left(w_{1}^{c}, x_{1}^{c}\right)$, with $w_{1}^{c}>\underline{w}$ and $x_{1}^{c}=0$; in state $\theta=\bar{\theta}$, the employer plays $\left(w_{\bar{\theta}}^{c}, x_{\bar{\theta}}^{c}\right)$, where $w_{\bar{\theta}}^{c}=\underline{w}$ and $x_{\bar{\theta}}^{c}=0$.

(ii) They play $\sigma^{c}$ again in period $t+1$ if $\left(w_{1}^{c}, x_{1}^{c}\right)$ was played in period $t$. If $\left(w_{\bar{\theta}}^{c}, x_{\bar{\theta}}^{c}\right)$ is played in period $t$, then, starting from period $t+1$, the union plays $(\widehat{e}, \widehat{s})$, where $\widehat{e}=1$, 
and $\widehat{s} \geq 0$; the employer plays $(\widehat{w}, \widehat{x})$, where $\widehat{w}>\underline{w}$, and $\widehat{x}=0$ for all $\theta$, during $T$ periods. Both players return to cooperation and play $\sigma^{c}=\left(e^{c}, s^{c}, w_{\theta}^{c}, x_{\theta}^{c}\right)$ in period $t+1+T$.

(iii) If the union plays $\left(e^{\prime}, s^{\prime}\right) \neq\left(e^{c}, s^{c}\right)$ in a period $t$ such that $\left(e^{c}, s^{c}\right)$ is required, or if the union plays $\left(e^{\prime}, s^{\prime}\right) \neq(\widehat{e}, \widehat{s})$ in a period $t$ such that $(\widehat{e}, \widehat{s})$ is required (i.e., during a punishment spell), then in period $t+1$, both players return to the noncooperative equilibrium $\sigma^{*}$ of $G$ during $T^{*}$ periods, after which they return to cooperative play $\sigma^{c}$, in period $t+T^{*}+1$.

(iv) If the employer plays $\left(w^{\prime}, x^{\prime}\right)$ such that $\left(w^{\prime}, x^{\prime}\right) \neq\left(w_{\bar{\theta}}^{c}, x_{\bar{\theta}}^{c}\right)$ and $\left(w^{\prime}, x^{\prime}\right) \neq\left(w_{1}^{c}, x_{1}^{c}\right)$, in a period $t$, such that $\left(w^{c}, x^{c}\right)$ is required, or if the employer plays $\left(w^{\prime}, x^{\prime}\right) \neq(\widehat{w}, \widehat{x})$ in $a$ period $t$ such that $(\widehat{w}, \widehat{x})$ is required, then, starting from period $t+1$, both players return to the noncooperative equilibrium $\sigma^{*}$ of $G$ in period $t+1$, during $T^{*}$ periods, after which they return to cooperation and play $\sigma^{c}$ in period $t+T^{*}+1$.

Using a terminology introduced by Athey and Bagwell (2001), there are "off-schedule" and "on-schedule" deviations of the employer. The on-schedule deviation is not observable: such deviations happen if the firm plays $\left(w_{1}^{c}, x_{1}^{c}\right)$ when the state is bad or plays $\left(w_{\bar{\theta}}^{c}, x_{\bar{\theta}}^{c}\right)$ when the state is good. Any choice $(w, x)$, different from $\left(w_{1}^{c}, x_{1}^{c}\right)$ or $\left(w_{\bar{\theta}}^{c}, x_{\bar{\theta}}^{c}\right)$ is necessarily detected as a deviation by the union when the game is in a cooperative phase, and any $(w, x)$ different from the required $(\widehat{w}, \widehat{x})$ is also detected, during punishement phases. These deviations are off-schedule. Union deviations can only be off-schedule. We must now check that our equilibrium candidate satisfies all incentive constraints for a nonnegligible set of parameter values.

\subsubsection{Value functions}

Let $V$ be the long-run expected utility of the employer along any path of play induced by our candidate equilibrium. Let $V_{1}$ be the expected, discounted utility when the state is $\theta=1$, and let $V_{\theta}$ be the expected, discounted utility when the state is $\bar{\theta}>1$. Recalling that $\pi$ is the probability of a good state, we have,

$$
V=\pi V_{1}+(1-\pi) V_{\theta}
$$

If $\theta=1$, then $V_{1}$ satisfies the equation,

$$
V_{1}=(1-\delta) v_{1}^{c}+\delta V
$$


where $v_{1}^{c}$ is the one-shot utility of the cooperative play $\sigma^{c}$, and we denote $v_{1}^{c}=v_{1}\left(e^{c}, s^{c}, w_{1}^{c}, x_{1}^{c}\right)$.

Next, if the true value of the state is $\bar{\theta}$, the employer plays $x_{\bar{\theta}}^{c}=0$ and $w_{\bar{\theta}}^{c}=1$, this triggers a punishment spell lasting $T$ periods in the period immediately following a bad draw of $\theta$; then, $\widehat{\sigma}$ is played $T$ times; the per-period expected utility of the employer is $\widehat{v}$ in each of these periods, and this latter term must be weighted by the annuity factor:

$$
\delta+\delta^{2}+\ldots+\delta^{T}=\delta \frac{\left(1-\delta^{T}\right)}{1-\delta} .
$$

Finally, the players return to cooperation at the end of the punishment phase. This has an expected, discounted value $\delta^{T+1} V$. It follows that $V_{\theta}$ satisfies the equation,

$$
V_{\theta}=(1-\delta) v \bar{\theta}+\delta\left(1-\delta^{T}\right) \widehat{v}+\delta^{T+1} V,
$$

where $v_{\bar{\theta}}^{c}=v_{\bar{\theta}}\left(e^{c}, s^{c}, w_{\bar{\theta}}^{c}, x_{\bar{\theta}}^{c}\right)$. Using the linear equations defining the value functions above, we easily obtain,

$$
V=\widehat{v}+\frac{(1-\delta)}{\Delta}\left(v^{c}-\widehat{v}\right),
$$

where, to simplify notation, we denote $v^{c}=E v_{\theta}^{c}$ and

$$
\Delta=1-\delta \pi-(1-\pi) \delta^{T+1} .
$$

Since $(1-\pi) \delta^{T+1}<1-\pi<1-\delta \pi$, we easily conclude that $\Delta>0$. The union's expected utility and value functions have parallel definitions; it is sufficient to replace $V$ with $U$ and $v$ with $u$ in the above formulas to find $U$.

\subsubsection{Incentive constraints: off-schedule deviations}

We first check a number of incentive constraints, due to the possibility of off-schedule deviations. The employer can play an unexpected $\left(w^{\prime}, x^{\prime}\right)$ in state $\theta$. Given that such a deviation will trigger a reversion to the Nash equilibrium of $G$ during $T^{*}$ periods, these deviations are deterred if and only if,

$$
V_{\theta} \geq(1-\delta) v_{\theta}^{\prime}+\delta\left(1-\delta^{T^{*}}\right) v^{*}+\delta^{T^{*}+1} V,
$$

where $v_{\theta}^{\prime}=\max _{(w, x)} v_{\theta}\left(e^{c}, s^{c}, w, x\right)$, for all $\theta$. This will always be true if $T^{*}$ is large enough and $\delta$ is sufficiently close to 1 . To see this, remark first that if $\theta=\bar{\theta}$, substituting the 
expressions for $V$ and $V_{\theta}$ in $\mathrm{IC}_{0 \bar{\theta}}$, we obtain,

$$
0 \geq(1-\delta)\left(v_{\bar{\theta}}^{\prime}-v_{\bar{\theta}}^{c}\right)-\delta\left(1-\delta^{T^{*}}\right)\left(\widehat{v}-v^{*}\right)-\frac{\delta(1-\delta)}{\Delta}\left(\delta^{T}-\delta^{T^{*}}\right)\left(v^{c}-\widehat{v}\right),
$$

and this inequality holds if $T^{*}$ is sufficiently large and $\delta$ sufficiently close to 1 . For instance, take $T^{*}=+\infty$, then, the inequality is true for all $\delta$ greater than some $\delta_{0}<1$, because $v^{*}<\widehat{v}$ and $\lim _{\delta \rightarrow 1}(1-\delta) / \Delta=(1+T(1-\pi))^{-1}<\infty$. If $\theta=1$, substituting the expressions for $V$ and $V_{1}$ in $\mathrm{IC}_{01}$, we obtain a similar result.

The union's deviation can only be off-schedule. It can be shown that these deviations can be deterred by reversion to $\sigma^{*}$ during $T^{*}$ periods too. We can state the following result.

Result 4. There exists values $\delta_{0}<1$ and $T_{0}>0$, such that for all $\delta$ in the open interval $\left(\delta_{0}, 1\right)$ and all $T^{*}>\max \left(T_{0}, T\right)$, the constraints $I C_{0 \theta}$ hold as strict inequalities and the off-schedule deviations of both players are not profitable.

The proof of Result 4 is completed in Appendix B.

\subsubsection{Incentive constraints: honesty and guts}

We now consider on-schedule deviations of the employer. In the good state, the employer should have no incentive to act as if the state was bad. In other words, the employer should be "honest". This imposes,

$$
V_{1} \geq(1-\delta) \widetilde{v}_{1}^{c}+\delta\left(1-\delta^{T}\right) \widehat{v}+\delta^{T+1} V
$$

where by definition, $\widetilde{v}_{1}^{c}$ is the one-shot payoff of playing $\left(w_{\bar{\theta}}^{c}, x_{\bar{\theta}}^{c}\right)$ when $\theta=1$, or $\widetilde{v}_{1}^{c}=$ $v_{1}\left(e^{c}, s^{c}, w_{\bar{\theta}}^{c}, x_{\bar{\theta}}^{c}\right)$. We easily get $\widetilde{v}_{1}^{c}=v_{0}-1$. A punishment phase starts immediately after this deviation. Using $V_{1}=(1-\delta) v_{1}^{c}+\delta V$, constraint $I C_{1}$ can be rewritten,

$$
\delta\left(1-\delta^{T}\right)(V-\widehat{v}) \geq(1-\delta)\left(\widetilde{v}_{1}^{c}-v_{1}^{c}\right)
$$

Now, using the expression for $V$ above, we easily derive the following equivalent form of $I C_{1}$,

$$
\frac{\delta\left(1-\delta^{T}\right)}{\Delta}\left(v^{c}-\widehat{v}\right) \geq \widetilde{v}_{1}^{c}-v_{1}^{c}
$$


The appropriately discounted value of the expected incremental benefits from cooperation must be greater than or equal to the temporary benefits of behaving as if the cost was high when it is in fact low.

The employer must also prefer to pay a low wage instead of hiding the bad news that the cost is high to avoid the punishment phase. In other words, the employer should have the guts to face conflict. Formally, we must have,

$$
V_{\theta} \geq(1-\delta) \widetilde{v}_{\bar{\theta}}^{c}+\delta V
$$

where $\widetilde{v}_{\bar{\theta}}^{c}$ is the payoff of playing $\left(w_{1}^{c}, x_{1}^{c}\right)$ when $\theta>1$, or $\widetilde{v}_{\bar{\theta}}^{c}=v_{\bar{\theta}}\left(e^{c}, s^{c}, w_{1}^{c}, x_{1}^{c}\right)$. We easily get $\widetilde{v}_{\bar{\theta}}^{c}=v_{0}-\bar{\theta} w_{1}^{c}$. The employer incurs a cost while deviating in this way - because the union receives a high wage $w_{1}^{c}>1$, but they avoid the punishment phase. Using the expression $V_{\theta}=(1-\delta) v_{\bar{\theta}}^{c}+\delta\left(1-\delta^{T}\right) \widehat{v}+\delta^{T+1} V$, it is easy to check that $I C_{2}$ is equivalent to

$$
v_{\bar{\theta}}^{c}-\widetilde{v}_{\bar{\theta}}^{c} \geq \frac{\delta\left(1-\delta^{T}\right)}{\Delta}\left(v^{c}-\widehat{v}\right) .
$$

To simplify notation, define

$$
\varphi(\delta, \pi, T)=\frac{\delta\left(1-\delta^{T}\right)}{1-\delta \pi-(1-\pi) \delta^{T+1}} .
$$

The incentive constraints $I C_{1}$ and $I C_{2}$, for "honesty" and "guts" respectively, can be rewritten as

$$
v_{\bar{\theta}}^{c}-\widetilde{v}_{\bar{\theta}}^{c} \geq \varphi(\delta, \pi, T)\left(v^{c}-\widehat{v}\right) \geq \widetilde{v}_{1}^{c}-v_{1}^{c} .
$$

Using the expressions for $\widehat{v}, v^{c}, \widetilde{v}_{1}^{c}, v_{1}^{c}, v_{\bar{\theta}}^{c}$ and $\widetilde{v} \frac{c}{\theta}$, and the definition of the bonus paid in the good state, $b=w_{1}^{c}-1$, we easily get that $I C_{12}$ is equivalent to $b \leq \varphi(\delta, \pi, T)[\widehat{A}-\pi b] \leq \bar{\theta} b$, where by definition, $\widehat{A}=\widehat{s} v_{0}+\mu \widehat{w}(1-\widehat{s})-\mu$. The constraints $I C_{12}$ can finally be rearranged as in the following statement ${ }^{22}$.

\section{Result 5.}

$I C_{12}$ is equivalent to the following string of inequalities,

$$
\frac{\varphi \widehat{A}}{\bar{\theta}+\pi \varphi} \leq b \leq \frac{\varphi \widehat{A}}{1+\pi \varphi},
$$

\footnotetext{
${ }^{22}$ Since $v^{c}-\widehat{v}=\widehat{A}-\pi b$, we can also rewrite constraint $\mathrm{IC}_{12}$ in compact form as $b \leq \varphi\left(v^{c}-\widehat{v}\right) \leq \bar{\theta} b$. In addition, since $\widehat{A}=\widehat{s}\left(v_{0}-\mu \widehat{w}\right)+\mu(\widehat{w}-1)$, and given that $\widehat{w}>1$, and $\widehat{v}=v_{0}-\mu \widehat{w}>0$, it is easy to see that $\widehat{A}>0$.
} 
where $\widehat{A}=\widehat{s} v_{0}+\mu \widehat{w}(1-\widehat{s})-\mu>0$

It remains to show that the set of parameter values for which "honesty" and "guts" hold is non-empty (and non-negligible).

\subsection{Main results}

Individual rationality constraints must also hold: we must have $u^{c}>\widehat{u}$ and $v^{c}>\widehat{v}$. Given the expressions of these terms, this is equivalent to $\pi b>(\widehat{w}-1)$ and $\mu(\widehat{w}-1)>\pi b$. Hence, we have the additional constraints,

$$
\frac{(\widehat{w}-1)}{\pi}<b<\frac{\mu(\widehat{w}-1)}{\pi}
$$

In the Appendix, we prove that the intersection of $\mathrm{IC}_{12}$ and $\mathrm{IR}_{12}$ is nonempty if $\bar{\theta}$ is large enough. More precisely, there exists a nonempty open interval $\left(b^{\min }, b^{\max }\right)$ of values of the bonus $b$, such that $\mathrm{IC}_{12}$ and $\mathrm{IR}_{12}$ hold simultaneoulsy. This interval can be defined as follows:

$$
b^{\min }=\max \left\{\frac{\varphi \widehat{A}}{\bar{\theta}+\pi \varphi}, \frac{(\widehat{w}-1)}{\pi}\right\}<b<\min \left\{\frac{\varphi \widehat{A}}{1+\pi \varphi}, \frac{\mu(\widehat{w}-1)}{\pi}\right\}=b^{\max } .
$$

We can therefore state,

\section{Result 6.}

Under Assumptions 1-4, for all $\delta$ in $(0,1)$ and $T \geq 1$, there exists a threshold $\bar{\theta}_{0}$, defined as

$$
\bar{\theta}_{0}=1+\frac{1}{\pi(1-\pi) \varphi}
$$

and an upper bound $s_{0}>0$, such that, for all $\bar{\theta}>\bar{\theta}_{0}$ and $\widehat{s}<s_{0}$, the values of $w_{1}^{c}$ picked in the interval $\left[1+b^{\min }, 1+b^{\max }\right]$ simultaneously satisfy the constraints $I C_{12}$ and $I R_{12}$ and $b^{\min }<b^{\max }$ : the interval is nonempty. In particular, on-schedule deviations are not profitable on this interval.

The proof of Result 6 is completed in Appendix B.

Note that when $\delta \rightarrow 0$, then $\varphi \rightarrow 0$ and $b^{\max } \rightarrow 0$ : the interval shrinks and vanishes. We therefore need patient players, but we also need $\bar{\theta}$, the "cost of funds" in the bad state, to 
be high enough. It is easy to check that,

$$
\lim _{\delta \rightarrow 1} \lim _{T \rightarrow \infty}(1-\pi) \varphi=1 .
$$

It follows that when $T$ is large and players are very patient, we require no more than $\bar{\theta}>$ $1+1 / \pi$, and if $\pi$ is itself close to 1 , this means approximately $\bar{\theta}>2$. With a sufficiently large $\bar{\theta}$, the inefficiencies induced by the rigid wage $\widehat{w}$ are nonnegligible, and this enlarges the set of feasible solutions.

Taken together, Results 1-6 show that our equilibrium candidate (defined above), is a PPE of $G(\delta)$ for sufficiently patient players. The following proposition summarizes our result.

Proposition 1. Under Assumptions 1-4, let $\widehat{w}$ belong to the nonempty interval $u^{*}+1<$ $\widehat{w}<\left(v_{0}-v^{*}\right) / \mu$. Then, for all $T \geq 1$, there exist real numbers $\delta_{0}<1, \beta_{0}>0, s_{0}>0, \bar{\theta}_{0}>1$, an integer $T_{0}>0$ and a nonempty interval of wages $\left[1+b^{\min }, 1+b^{\max }\right]$ (defined above) such that, for all $1>\delta>\delta_{0}, \beta>\beta_{0}, \widehat{s}<s_{0}, T^{*}>T_{0}, \bar{\theta}>\bar{\theta}_{0}, 1+b^{\min }<w_{1}^{c}<1+b^{\max }$, the equilibrium candidate (defined above) is a Perfect Public Equilibrium of $G(\delta)$.

The result describes conditions under which there exists an equilibrium characterized by an "ordinary course of business" in which the union and the employer cooperate, but such that finite-length, inefficient-conflict spells are triggered by the random occurrence of a high-cost state. During these conflict spells, the employees' wage is kept above its minimum, the employees' effort is still high and there is no outsourcing, but the probability of strikes is positive, i.e., a nonzero fraction of output will be lost, due to work stoppages, during each of these periods. The cooperative phases are characterized by high wages, high effort on the part of employees, zero outsourcing and no strikes ${ }^{23}$.

Slowdown and strikes are in a certain sense substitutes as a way of "burning money". An equilibrium of the same type, in which strikes disappear but slowdown, outsourcing and low wages characterize punishment phases also exists. It is easy to construct a PPE

\footnotetext{
${ }^{23}$ The occurrence of strikes - as well as the low-effort spells - is entirely due to the presence of informational asymmetries. The threat of a return to non-cooperative equilibrium in which strike activity is zero, but outsourcing is positive, while effort and wages are low plays a crucial role, but lies off the equilibrium path.
} 
of this sort in our model, using reversion to $\sigma^{*}$, instead of $\widehat{\sigma}$, when a bad state of nature is drawn. In the definition of the equilibrium candidate, we replace $\widehat{\sigma}=(\widehat{e}, \widehat{s}, \widehat{w}, \widehat{x})$ with $\sigma^{*}=\left(e^{*}, s^{*}, w^{*}, x^{*}\right)$ everywhere and obtain a valid statement as follows.

Proposition 2. In the definition of the equilibrium candidate, replace everywhere $\widehat{\sigma}=$ $(\widehat{e}, \widehat{s}, \widehat{w}, \widehat{x})$ with $\sigma^{*}=\left(e^{*}, s^{*}, w^{*}, x^{*}\right)$. Then, under Assumptions $1-4$, for all $T \geq 1$, there exist real numbers $\delta_{0}<1, \beta_{0}>0, \underline{e}_{0}<1$, an integer $T_{0}>0$ and a nonempty interval of wages $\left[1+b^{\min }, 1+b^{\max }\right]$ such that, for all $1>\delta>\delta_{0}, \beta>\beta_{0}, \underline{e}_{0}<\underline{e}<1, T^{*}>T_{0}$, $1+b^{\min }<w_{1}^{c}<1+b^{\max }$, the equilibrium candidate in which $\widehat{\sigma}$ is everywhere replaced with $\sigma^{*}$ is a Perfect Public Equilibrium of $G(\delta)$.

Proposition 2 is proved in Appendix B.

\subsection{Inefficiency of Equilibria}

Finally, can we find a fully efficient equilibrium? In other words, does there exist equilibria such that the expected total average utility of the players is equal to the highest possible value of surplus, namely, such that $U+V=u^{c}+v^{c}$ ? For any given value of $\delta<1$, the answer to this question is no. To prove this assertion, remark first that in our model, utility can be freely transfered only in the good state of nature $\theta=1$. In this state, the slope of the utility possibility frontier is -1 : any unit of value taken from the employer's pocket is worth exactly one unit for the union. The efficient frontier is defined by $x_{\theta}=0, s=0$, $e=1$, and $w_{\bar{\theta}}=1$. A fully efficient equilibrium cannot possess a path which drops below the efficient frontier with a positive probability. An efficient equilibrium path should therefore remain on the efficient frontier in all periods and $w_{1}$ is the only variable that can vary during fully efficient equilibrium play. This means that increases in the bonus $b=w_{1}-1$ are the only way of punishing the firm. The firm can be punished for on-schedule deviations only if the state is good, but the state is not observed by the union. Hence, whichever the union's decision rule to trigger punishments, the firm can always claim that the state is bad, i.e., propose the minimum wage, and avoid any punishment ${ }^{24}$. It follows that the only possible

\footnotetext{
${ }^{24}$ For instance, the union could behave as a statistician, and compute the frequency of periods during which the wage is not equal to its minimum. When this frequency is below $\pi$ with a sufficient degree of significance,
} 
fully-efficient equilibria must be such that the wage is constantly equal to its minimum, or the bonus $b=0$. The union's payoff in a fully efficient equilibrium candidate is therefore $(1-\bar{e})=0$, but this is the union's $\operatorname{minmax}^{25}$ in $G$. Hence, if the candidate-equilibrium's union payoff is strictly above 0 there must exist one state at least in which $w_{\theta}>1=\underline{w}$. We can state the following result.

Proposition 3. If $\delta<1$, all PPE such that the union's payoff is strictly positive are inefficient. In other words, if in a given PPE, the employer pays a wage higher than the minimum, i.e., $w_{\theta}>\underline{w}$, with a positive probability, then, its equilibrium payoffs $(U, V)$ are below the efficient frontier: $U+V<u^{c}+v^{c}$.

\subsection{Constrained Efficiency}

We would like to know if our family of equilibria is in a sense second-best efficient. To this end, we first define a notion of second-best, or constrained, incentive-efficient payoff in the static game $G$. We then show that the candidate equilibria studied above can do better than the static second-best efficient level of surplus.

Consider now the one-shot game $G$ as a static mechanism design problem. We will search for the best direct, incentive-compatible revelation mechanism $\theta \rightarrow\left(e_{\theta}, s_{\theta}, w_{\theta}, x_{\theta}\right)$. In this mechanism, the informed agent is the employer, the principal is the union and we apply the Revelation Principle (on these notions, see Laffont and Martimort (2001)). Define $h_{\theta}=1$ if $e_{\theta}=1$ and $h_{\theta}=\bar{h}$ if $e_{\theta}=\underline{e}$. The employer reports a state $\theta$ and the "planner" imposes $\left(e_{\theta}, s_{\theta}, w_{\theta}, x_{\theta}\right)$. Second-best optima are solutions of the following optimization problem:

$$
\text { maximize } E\left\{h_{\theta}\left(1-s_{\theta}\right)\left(1-x_{\theta}\right)\left(w_{\theta}-e_{\theta}\right)\right\}
$$

the union can try to punish the firm by increasing $w_{1}$, and revert to the Nash equilibrium of $G$ if the firm doesn't comply. But then, the firm can continue to cheat forever if no other change is made. At some point, if the observed frequency of high-pay periods is significantly below $\pi$, the union has no other choice but to punish the employer by choosing inefficient values of $s$ and (or) $e$ and (or) by changing the required $w_{\theta}$ in all states $\theta$, and with a positive probability at least. Given that this strategy relies on a statistical test, the wrong decision will be made by the union with some positive probability and the equilibrium payoffs will therefore lie below the efficient boundary.

${ }^{25}$ Note that the union's minmax is obtained when the employer plays $x=1$. 
subject to the constraints,

$$
E\left\{v_{0}\left(1-s_{\theta}\left(1-x_{\theta}\right)\right)-\theta A_{\theta}\right\} \geq \underline{v}
$$

and

$$
\begin{aligned}
v_{0}\left(1-s_{\bar{\theta}}\left(1-x_{\bar{\theta}}\right)\right)-\bar{\theta} A_{\bar{\theta}} & \geq v_{0}\left(1-s_{1}\left(1-x_{1}\right)\right)-\bar{\theta} A_{1}, \\
v_{0}\left(1-s_{1}\left(1-x_{1}\right)\right)-A_{1} & \geq v_{0}\left(1-s_{\bar{\theta}}\left(1-x_{\bar{\theta}}\right)\right)-A_{\bar{\theta}}
\end{aligned}
$$

where by definition,

$$
A_{\theta}=h_{\theta}\left(1-s_{\theta}\right)\left(1-x_{\theta}\right) w_{\theta}+C\left(x_{\theta}\right)
$$

and $\underline{v}$ is a parameter.

Note that $\mathrm{ICa}$ and $\mathrm{ICb}$ together can be rewritten as follows,

$$
A_{1}-A_{\bar{\theta}} \leq v_{0}\left(s_{\bar{\theta}}\left(1-x_{\bar{\theta}}\right)-s_{1}\left(1-x_{1}\right)\right) \leq \bar{\theta}\left(A_{1}-A_{\bar{\theta}}\right) .
$$

This string of inequalities implies $A_{1}-A_{\bar{\theta}} \geq 0$, thus $s_{\bar{\theta}}\left(1-x_{\bar{\theta}}\right) \geq s_{1}\left(1-x_{1}\right)$. First-best efficiency implies $s_{\theta}=x_{\theta}=0$ and $e_{\theta}=1$, and in the bad state, $w_{\bar{\theta}}=1$. From this we find that $I C a b$ implies $0 \leq w_{1}-1 \leq 0 \leq \bar{\theta}\left(w_{1}-1\right)$. Thus, the only fully efficient and incentive compatible solution entails $w_{1}=w_{\bar{\theta}}=1$ and $E\left(u_{\theta}\right)=0$. If we look for a second-best allocation such that $w_{1} w_{\bar{\theta}}>1$, we must solve the above optimization problem. Standard methods can be applied. It can be shown that $I C b$ and $I R$ are binding at the optimum if $v_{0}$ is large enough. Let $\left(\widetilde{e}_{\theta}, \widetilde{s}_{\theta}, \widetilde{w}_{\theta}, \widetilde{x}_{\theta}\right)$ denote the second-best solution. Let $\widetilde{u}$ and $\widetilde{v}$ denote the second-best optimal expected payoffs and $\widetilde{R}=\widetilde{u}+\widetilde{v}$ denote the second-best surplus. Then, we have the following result.

Result 7. Under Assumptions 1-4, if $v_{0}$ is sufficiently high, in a second-best optimal, incentive-compatible revelation mechanism, the employer pays a wage higher than the minimum in some state, i.e., $\widetilde{w}_{1} \widetilde{w}_{\bar{\theta}}>1, I R$ and $I C b$ are binding and we have, $\widetilde{e}_{1}=\bar{e}=1$; $\widetilde{s}_{1}=\widetilde{x}_{1}=0$.

There are three cases, with different solutions in the bad state, and two different expressions of payoffs: 
(i), if $v_{0}(1-\pi(\bar{\theta}-1))<\mu$, work stops completely in the bad state, namely, $\widetilde{s}_{\bar{\theta}}=1$; and outsourcing takes place, more precisely, $\widetilde{x}_{\bar{\theta}}>0$ is a feasible solution of the equation $\underline{v}=$ $\left(v_{0}-\mu\right) \widetilde{x}_{\bar{\theta}}+\beta \mu \widetilde{x}_{\bar{\theta}}^{2}$; the wage paid in the good state is given by the expression

$$
\widetilde{w}_{1}=(1 / \pi)\left[v_{0}\left(1-(1-\pi)\left(1-\widetilde{x}_{\bar{\theta}}\right)\right)-\underline{v}-(1-\pi) \bar{\theta} C\left(\widetilde{x}_{\bar{\theta}}\right)\right]
$$

the second-best optimal expected payoffs are

$$
\widetilde{u}=\pi\left(v_{0}-1\right)-(1-\pi)\left[v_{0} \widetilde{x}_{\bar{\theta}}+\bar{\theta} C\left(\widetilde{x}_{\bar{\theta}}\right)\right]-\underline{v} \quad \text { and } \quad \widetilde{v}=\underline{v} .
$$

(ii), if $v_{0}(1-\pi(\bar{\theta}-1)) \geq \mu \underline{\underline{h}}$, then, there are no strikes and no outsourcing, $\widetilde{s}_{\bar{\theta}}=\widetilde{x}_{\bar{\theta}}=0$; $\widetilde{e}_{\bar{\theta}} \widetilde{h}_{\bar{\theta}}=1$; the wage is constant, above the minimum, $\widetilde{w}_{\bar{\theta}}=\widetilde{w}_{1}=\left(v_{0}-\underline{v}\right) / \mu>1$; the secondbest optimal expected payoffs are

$$
\widetilde{u}=-1+\left(v_{0}-\underline{v}\right) / \mu \quad \text { and } \quad \widetilde{v}=\underline{v} .
$$

(iii), if $\mu \underline{\underline{e}} \bar{h}>v_{0}(1-\pi(\bar{\theta}-1)) \geq \mu$, the solution is either the same as in case (ii) above, with $\widetilde{e}_{\bar{\theta}}=1$, and $\widetilde{s}_{\bar{\theta}}=0=\widetilde{x}_{\bar{\theta}}$, or the same as in case $(i)$ above, with $\widetilde{e}_{\bar{\theta}}=\underline{e}$, and $\widetilde{s}_{\bar{\theta}}=1$.

The proof of Result 7 is in Appendix B.

Intuitively, there are several ways of burning money. Result 7 shows that money can be burnt by means of strikes and outsourcing if the probability of bad states is small, since in this case, strikes are relatively cheaper. In contrast, if the probability of bad states is relatively high, it is more efficient to use a rigid wage system, since a high wage in the bad state is then a less destructive way of wasting resources. We conclude the formal analysis of our model by showing that in the repeated game, expected surplus can be greater than in the static second-best optimal mechanism, using the strategies described above as our equilibrium candidate.

Result 8. Under Assumptions 1-4, if $v_{0}$ is large enough, then, there exists feasible pairs $(\widehat{s}, \widehat{w})$ such that $R>\widetilde{R}$. In other words, the equilibrium strategies defined above as our equilibrium candidate support payoffs such that their sum is greater than the second-best surplus $\widetilde{R}$, for appropriately chosen values of $(\widehat{s}, \widehat{w})$. 
The proof of Result 8 is given in Appendix B.

To sum up, we have been able to show that our candidate equilibrium is indeed and equilibrium (Proposition 1), that slowdown can replace strikes as a means of burning resources (Proposition 2) and finally, that if our candidate equilibrium can do at least as well as the second-best, incentive efficient level of surplus in the one-shot game $G$ (Result 8 ), for $\delta<1$, all equilibria must be first-best inefficient.

\section{Interpretations}

We now study some properties of the model and discuss their ability to shed light on relationships between facts.

\subsection{Minimal length of the punishment phase}

Our equilibrium candidate supports an expected discounted value of the surplus denoted $R=U+V$. Given the expressions of $U$ and $V$, we have,

$$
R=\widehat{u}+\widehat{v}+\frac{(1-\delta)}{\Delta}\left(u^{c}+v^{c}-\widehat{u}-\widehat{v}\right)
$$

Remark that $u^{c}, v^{c}, \widehat{u}$ and $\widehat{v}$ do not depend on $T$. If we now treat $T$ as a real number, we find that $(1-\delta) / \Delta$ is a decreasing function of $T$. It follows from this that $\partial R / \partial T<0$ and the optimal value of $T$ is therefore the smallest value compatible with $\mathrm{IC}_{12}$ and $\mathrm{IR}_{12}$. Now, going back to $\mathrm{IC}_{12}$, we find that $\varphi$ is a strictly increasing function of $T$. As a consequence, for given values of $b$ and $(\widehat{s}, \widehat{w}), \mathrm{IC}_{1}$ will be binding at the smallest real admissible value of

$T$, denoted $\widetilde{T}$; that is, we will have $\varphi(\delta, \pi, T)\left(v^{c}-\widehat{v}\right)=b$ or equivalently, $b=b^{\max }$. We summarize a number of findings in the following statement.

Result 9. The real solution of the equation $\varphi(\delta, \pi, T)\left(v^{c}-\widehat{v}\right)=b$ with respect to $T$, denoted $\widetilde{T}$, is a well-defined and differentiable function of $\left(\delta, \pi, v_{0}, b, \widehat{w}, \widehat{s}\right)$. The minimal value of $T$, an integer, is $T^{\mathrm{min}}=\operatorname{int}(\widetilde{T})+1$, where $\operatorname{int}(T)$ is the largest integer smaller than or equal to $\widetilde{T}$. The following properties hold:

$$
\frac{\partial}{\partial T}\left(\frac{1-\delta}{\Delta}\right)<0 ; \quad \frac{\partial \varphi}{\partial T}>0 ; \quad \frac{\partial \widetilde{T}}{\partial v_{0}}<0 ; \quad \frac{\partial \widetilde{T}}{\partial b}>0 ; \quad \frac{\partial \widetilde{T}}{\partial \widehat{s}}<0 ; \quad \frac{\partial \widetilde{T}}{\partial \widehat{w}}<0
$$


The minimal admissible value of $\left(v^{c}-\widehat{v}\right)$ is $b(1-\delta \pi) / \delta$. When $\left(v^{c}-\widehat{v}\right)$ approaches this minimal value, then $\widetilde{T} \rightarrow+\infty$. Hence, when $\widehat{s}$ become so small that $\left(v^{c}-\widehat{v}\right)$ approaches its minimal value, then $\widetilde{T}$ tends toward infinity.

For proof, see Appendix B.

The minimal value $T^{\text {min }}$ is a function of $b, \widehat{w}$ and $\widehat{s}$. We now discuss some comparative statics properties of $\widetilde{T}$. Given that $\varphi \eta=b$, with $\eta=v^{c}-\widehat{v}$ and $\varphi$ is increasing in $T$, we see that $\widetilde{T}$ must be decreasing in $\eta$, and calculus confirms that $\partial \widetilde{T} / \partial \eta<0$. An increase in the "instantaneous surplus of cooperation" $\eta$ decreases the minimal length of the punishment phase - an easily understandable property. From this we derive,

$$
\frac{\partial \widetilde{T}}{\partial v_{0}}=\widehat{s} \frac{\partial \widetilde{T}}{\partial \eta}<0 .
$$

An increase in the value of production reduces the length of punishment phases all the more since strikes are long.

After some computations, we obtain, $\partial \widetilde{T} / \partial b>0$. There is a tradeoff between the prevalence of conflict and the wage rate obtained by workers in the cooperation phases. It must be that the fraction of time spent in a situation of conflict increases when the bonus is more generous. Increases in the bonus can only be sustained if the length of the minimal punishment phase is increased, because the employer's rewards from cooperation are reduced.

Another important tradeoff is between $\widehat{s}$ and $\widetilde{T}$. Easy calculus yields,

$$
\frac{\partial \widetilde{T}}{\partial \widehat{s}}=\left(v_{0}-\mu \widehat{w}\right) \frac{\partial \widetilde{T}}{\partial \eta}<0 .
$$

More strikes during punishment spells should therefore be associated with shorter such spells. This will generate a tradeoff between the duration and the incidence of strikes (we define incidence precisely below). In a cross-section of firms, we would observe that firms with longer strikes are also such that strikes are less prevalent. Finally, we obtain,

$$
\frac{\partial \widetilde{T}}{\partial \widehat{w}}=\mu(1-\widehat{s}) \frac{\partial \widetilde{T}}{\partial \eta}<0 .
$$

When the rigid wage rate paid to workers during punishment phases is higher, the length of these phases should be lower. In Appendix B, we also ask the following question: given $\widehat{w}$, $b$ and $\delta$, can we find an optimal combination $(T, \widehat{s})$ ? 


\section{$5.2 \quad$ Incidence of strikes}

We now turn to the comparative statics of strike incidence, and study the ability of our model to reproduce a number of empirical observations. The empirical literature has discussed the behavior of strikes during the business $\operatorname{cycle}^{26}$. One of the main findings of this literature is, with qualifications, that strikes have a procyclical incidence. For instance, Vroman (1989) finds the latter property with US data between 1957 and 1984 ${ }^{27}$. McConnell (1990) finds that strike incidence is highest in industries that are depressed relative to the rest of the economy but in regions with low unemployment. In France, the first difference in the logaggregate number of days lost to strikes is positively correlated with the first difference in growth rates: the coefficient of correlation is 0.4 during the period 1977-2004. So, it seems that we have a pro-cyclical incidence of strikes at the aggregate level.

In our context, incidence is the unconditional probability of observing a strike, or the average fraction of time spent on strike in equilibrium. It can be defined in a simple way as the probability, denoted $q$, that a period chosen at random belongs to a punishment phase, assuming that the length of punishment phases is minimal, set equal to $T^{\mathrm{min}}$. We thus define a lower bound, a notion of "minimal incidence". The duration of strikes is simply $\widehat{s}$. Given the length of punishment phases, and the probability of bad states $(1-\pi)$, we can express the probability of non-cooperation in period $t$ as follows:

$$
1-\operatorname{Pr}(\text { cooperation at } t)=\sum_{\tau=1}^{T}(1-\pi) \operatorname{Pr}(\text { cooperation at } t-\tau),
$$

because the agents do not cooperate in period $t$ if a punishment phase has started $\tau$ periods before, with $\tau \leq T$. In equilibrium, a punishment phase starts if the players were cooperating in $t-\tau$ and if a bad state has been drawn (with probability $1-\pi$ ). Given the stationary nature of the model, $\operatorname{Pr}($ cooperation at $t)=\operatorname{Pr}($ cooperation at $t-\tau)=1-q$. From this, we immediately derive, $q=(1-\pi)(1-q) T$, that is,

$$
q=\frac{(1-\pi) T}{1+(1-\pi) T}
$$

\footnotetext{
${ }^{26}$ Research on this topic has started a long time ago. See, for instance, on the US case, Hansen (1921), Rees (1952), Ashenfelter and Johnson (1969), Kennan (1986), Kennan and Wilson (1989), Card (1990).

${ }^{27}$ On the Canadian case, see Harrison and Stewart (1994). For a study of strikes in the UK (and further references), see e.g., Devereux and Hart (2008).
} 
Suppose now that we have a sample of identical firms playing the same Perfect Public Equilibrium and with states drawn independently: $q$ is then the probability of drawing a firm experiencing an inefficient conflict spell. But if random states are uncorrelated across firms, it is impossible to interpret the state of nature as a business-cycle fluctuation. At the macroeconomic level, we observe more strikes in the country during booms, whereas our model seems to predict that strikes are triggered by the occurrence of slumps. So, it seems that our model predicts a counter-cyclical incidence of strikes, but this would be a naive interpretation. Recall that, in our model, the state is by definition private information of the employers. It is reasonable to assume that a general state of boom or bust would on the contrary be common knowledge. To derive a microeconomic foundation for the strike aggregates, we need strategies that are contingent on the publicly observed macroeconomic state, while the firm's state $\theta$ remains a privately observed, idiosyncratic shock. Suppose that unions are more demanding during booms: this means that they expect higher bonuses during booms. As a consequence, a given bonus can trigger a strike during a boom, while it would be accepted without a murmur in a state of bust. Another possibility is that, when faced with a given wage or bonus, unions will go on strike with a smaller probability in a state of bust. If this is the case, more strikes will be triggered in firms that are relatively disadvantaged during booms, that is, in firms with a bad drawing of their private state $\theta$, while the economy is booming. Now, if the length $T$ of reversions to inefficient play is not too long, and if the publicly observed state of the macroeconomy is sufficiently persistent, a positive correlation of booms with the incidence of strikes will be generated. The same mechanism will also generate a lag of the aggregate strikes cycle. This is a relatively straightforward extension of our model, since strategies can be made dependent on the outcome of a public lottery in a simple way, but requires lengthy computations to be worked out in details. With this type of extension, it seems clear that strikes can be pro-cyclical in the aggregate, even if, in fact, they are always triggered by some disappointing event at the microeconomic level.

Finally, does the model provide us with an interpretation of the facts related to the Paris dustmen? In particular, can we explain the drop in strike activity that we observe on Fig. 1, from the mid-eighties until the early 2000s? We think that this sharp drop can be explained as a change of regime. An equilibrium in which strikes are used as a punishment 
device has disappeared and has been replaced by an equilibrium in which slowdown is used as a punishment. The number of observed days of strike has fallen to zero, while outsourcing appeared. This can be checked on Fig. 2, showing that the number of civil-service dustmen has also been decreasing from 1984 to 2000. This new equilibrium looks like a PPE in which the one-shot Nash equilibrium is used during inefficient punishment phases: unrecorded slowdown replaces observed work stoppages. This regime switching is the likely result of a sharp reduction in the value of $\beta$, the specific cost of outsourcing. A drop in $\beta$ captures the change of attitude of the town hall with respect to privatization of garbage-collection services. Indeed, we know that a change of policy regarding outsourcing took place in the late seventies, early eighties. In our model of strikes, a drop of $\beta$ is likely to destroy the established equilibrium regime described above in Proposition 1. This is because we have

$$
\frac{\partial U^{*}}{\partial \beta}>0, \quad \text { and } \quad \frac{\partial V^{*}}{\partial \beta}<0
$$

If $V^{*}$ increases enough, then it hits $\widehat{V}$ from below and the prevailing equilibrium is no longer feasible. Since $\widehat{V}=(1-\widehat{s})\left(v_{0}-\mu \widehat{w}\right)$, maintaining the old equilibrium requires $\widehat{s} \rightarrow 0$ or a drop in $\widehat{w}$ that may be infeasible. An equilibrium in which strikes disappear but slowdown, outsourcing and low wages characterize punishment phases is likely to replace the former arrangement. We have shown that it is easy to construct a PPE of this sort in our model, using reversion to $\sigma^{*}$ instead of $\widehat{\sigma}$ when a bad state of nature is drawn (see Proposition 2 above). In the latter equilibria, punishment phases are not easily observable, because slowdown phases are not recorded as strike days. We know from our case study of the Paris dustmen, and there is a lot of anecdotal evidence confirming these trends, that the recent period is one in which effort has been low (with for instance higher absenteeism) and worker resistance has been high (with many more work-to-rule or slowdown spells than before). Recent statistics of the French Ministry of Labour show that if the number of outright strikes has been decreasing in the past thirty years, in contrast, nonstandard forms of conflict, slowdown and work-to-rule have been everywhere on the rise (e.g., DARES (2009)). A consequence of the inessential nature of strikes is that their causal effect on wages, as well as the causal impact of business-cycle indicators on strike incidence will be hard to identify, since other unobservable forms of labor strife can also have an impact on wages. 


\section{Conclusion}

We have modeled the long-run relationship between an employer and his workers' union as a repeated mechanism design problem, in a repeated game with imperfect monitoring. The union does not observe firm profitability, drawn at random in every period. In this context, we have shown that cooperation can be sustained if players are sufficiently patient, in spite of informational asymmetries, at the cost of random reversions to inefficient ways of playing, during finite-duration spells, in which strikes, slowdown and worker replacement may appear. We focus on a class of Perfect Public Equilibria in which strikes are used to support equilibrium. Under cooperation, workers enjoy high wages (i.e., positive bonuses), no outsourcing (and therefore more employment); the firm avoids strikes completely and productivity is higher, due to high worker effort. The equilibrium path is characterized by alternating phases of cooperative and inefficient play involving strikes. Transitions are caused by random external profitability shocks, which themselves cause low pay or a zero bonus event. We showed that all the equilibria of our game are inefficient. Given incentive constraints, first-best efficiency can be reached only if wages are constantly equal to their minimum, implying a zero bonus, but the union's payoff is then equal to its minmax. However, Perfect Public Equilibria may support an expected surplus that is higher than the second-best incentive-efficient level, obtained by means of a direct revealing mechanism in the static constituent game. In the class of equilibria that we studied, we have derived the minimal length of punishment spells. Incentive constraints have been shown to imply that higher wages or larger bonuses should be positively correlated with longer strike durations - but longer strikes do not cause higher wages. Some empirical facts from the municipal garbage-collection industry have been used to illustrate the theory and show the interaction

of outsourcing (or worker replacement), wages and strikes in the long run. In particular, our model can explain why strikes may disappear in response to changes in the environment and be replaced by other, less easily observable forms of conflict. The proposed theory can be viewed as a synthesis of the theory of strikes with that of relational contracts. 


\section{Appendix A: Further Study of Paris Dustmen}

A municipal dustman's lifetime career is somewhat rigidly organized. There is a rigid wagescale divided in 10 grades. Seniority plays a major role in promotions: a dustman stays on each grade (or step) for two or three years (depending on the grade). Those who become foremen (i.e., "head of sanitation team") start to climb up a different ladder. But the future of those who belong to the rank-and-file until the end of their careers is entirely described by the 10 grades, on the same scale. The pay corresponding to each grade varies over time, because each negotiation with the union can lead to alterations of the ladder's overall height, and to a lesser extent, of the difference between steps. The real value of each grade can also vary over time, because of pay raises decided by the country's central government and applied to all members of the civil service, including municipal workers, and because of inflation. Figure 3 shows the real value of the 10 grades of a dustman's career over time, with the real value of the minimum wage as a point of comparison (at the bottom). An additional, eleventh grade has been created in the nineties. Each wage-scale curve is roughly parallel to others.

The effects of Mayor Chirac's tough outsourcing policy can easily be measured. In 1977, the dustmen of Paris seemed enjoyed a comfortable rent; they earned substantially more than the national minimum wage: a first-grade beginner, which is typically an unskilled worker, would earn $25 \%$ more than an equivalent private-sector, minimum-wage worker. This rent is the likely result of the numerous strikes of the late sixties and early seventies (in particular of the well-known 1968 events). Another striking feature is that in the long run, the real values of the first grade and minimum wages converged. Public sector dustmen have lost the wage-premium they enjoyed 30 years ago. This seems to be the combined result of inflation, which eroded the real values, and of town hall resistance to the union's claims. The process of return to long-run "equilibrium" wages has been gradual. During some years, particularly the years 1984 to 1990, the real value of the lowest grades has decreased, and the real value of the highest grades has decreased from 1986 to 1989. But none of these curves does in fact describe the evolution of an individual worker's wage, because seniority triggers automatic grade promotions. Figure 4 plots a simulation of a typical career, showing 
the real wage of a dustman, hired in 1978, starting in the first grade, and promoted using the seniority rules that used to be in force. This dustman's wage has an increasing trend, with some fluctuations (such as the visible dip between 1987 and 1989); short periods of stagnation are followed by upward jumps, due to automatic grade promotions. Figure 4 also shows the real minimum wage as a point of comparison. It is likely that individual, seniority-induced raises have eased the town hall's austerity policy, by making the real-value erosion of wages less painful for workers.

Given the pay scale and promotion rules, it is possible to compute the real value of a dustman's career at each point in time: it is the present discounted value of a dustman's real wages over an entire career cycle, evaluated at a given moment using the promotion and pay rules in force. In other words, these discounted wage sums are computed each year for a first-grade new recruit, under the myopic assumption that current seniority rules will not change and that the real value of each grade will remain constant over the entire career. Figure 5 plots the series of real career values, based on the dustman's real take-home pay (in 2004 euros) and a discount rate of $3 \%$. Figure 5 simultaneously shows a decreasing linear trend and the swings of the best-fit curve, using a fifth-degree polynomial.

Finally, we have tried some regressions involving the real value of a career and the number of strike days lost. The results obtained should of course be interpreted with caution, due to the small number of observations, and to possible endogeneity problems, but still, they are sufficiently suggestive to be presented here. Let $V_{t}$ be the value of a career in year $t$. Let $\Delta V_{t}=V_{t}-V_{t-1}$ be the first difference. Let $\Delta N_{t}$ be the yearly variation in the total workforce, and let $S_{t}$ be the number of days lost due to strikes in year $t$. Are variations in $V_{t}$ in a certain way correlated with strikes $S_{t}$ ? The first column in Table 1 shows a linear regression of $V$ on $S$ which is not yielding a significant coefficient. But in the second column, a regression of $\Delta V_{t}$ on $S_{t}$ and $\Delta N_{t}$ yields a significant positive coefficient on strikes. Yet these results are somewhat disappointing. Define next the career-value increase $\Delta V_{t}^{+}=\max \left\{0, \Delta V_{t}\right\}$. With this latter variable, we find an interesting regression. Column 4 in Table 1 reports the results of $\Delta V_{t}^{+}=a+b S_{t}+c \Delta N_{t}+\varepsilon$, which yields a significant and positive $b$ and a negative $c$. Second, define value drops as $\Delta V_{t}^{-}=\min \left\{0, \Delta V_{t}\right\}$. We then run the regression $S_{t}=\alpha+\beta \Delta V_{t-1}^{-}+\gamma \Delta N_{t-1}+\varepsilon$ and find that $\beta$ is significant and negative, 
while $\gamma$ is significant and positive. The results of the latter regression are reported in column 5 of Table 1. Lagged value drops seem to "cause" strikes, while workforce reductions seem to harness strikes.

\section{Appendix B: Proofs}

\section{Proof of Result 2.}

We substitute the value of $s^{c}=0$ in the expression for $x_{\theta}^{c}$ and we obtain,

$$
x_{1}^{c}(e, 0)=(1 / \beta)[e h-1]^{+}, \quad x_{\bar{\theta}}^{c}(e, 0)=(1 / \bar{\theta} \beta)[e h+(\bar{\theta}-1) h-\bar{\theta}]^{+} .
$$

We must now determine the cooperative effort level $e^{c}$. High effort yields $h=\underline{h}=1$, and we

obtain, $x_{\bar{\theta}}^{c}(\bar{e}, 0)=(1 / \bar{\theta} \beta)[\bar{e}-1]^{+}=0$, since $\bar{e} \leq 1$, and $x_{1}^{c}(\bar{e}, 0)=(1 / \beta)[\bar{e}-1]^{+}=0$. From these results, we derive,

$$
E\left(B_{\theta} \mid e=\bar{e}, s=0\right)=v_{0}-E\left[(\theta-1) w_{\theta}^{c}+\bar{e}\right]
$$

If effort is low, we have $h=\bar{h}>1$, and $x_{\theta}^{c}(\underline{e}, 0)$ can now be positive. Hence, we obtain,

$$
E\left(B_{\theta} \mid e=\underline{e}, s=0\right)=v_{0}-E\left\{\left[1-x_{\theta}^{c}(\underline{e}, 0)\right]\left[(\theta-1) \bar{h} w_{\theta}^{c}+\underline{e} \bar{h}\right]\right\}-E\left[\theta C\left(x_{\theta}^{c}(\underline{e}, 0)\right)\right] .
$$

Now, since $\theta C\left(x_{\theta}^{c}().\right)$ is nonnegative, a sufficient condition for high effort $\bar{e}$ to be optimal is therefore,

$$
E\left[(\theta-1) w_{\theta}^{c}+\bar{e}\right]<E\left\{\left[1-x_{\theta}^{c}(\underline{e}, 0)\right]\left[(\theta-1) \bar{h} w_{\theta}^{c}+\bar{h} \underline{e}\right]\right\}
$$

The left-hand side of this inequality doesn't depend on $\beta$. If $\beta$ is sufficiently high, $x_{\theta}^{c}$ is as small as desired and the inequality then holds, because, under Assumptions 1 and $2, \underline{e} \bar{h}>\bar{e}$ and $\bar{h}>1$. This proves the result.

Q.E.D.

\section{Proof of Result 4 .}

Given the above analysis of $\mathrm{IC}_{0 \theta}$, we must now consider off-schedule deviations of both players to end the proof. The union's deviation can only be off-schedule. Suppose that the 
state is $\theta=1$, then, the union would not deviate from $\left(e^{c}, s^{c}\right)$ in the next period if and only if,

$$
U_{1} \geq(1-\delta) u_{1}^{c}+\delta(1-\delta) u^{\prime}+\delta^{2}\left(1-\delta^{T^{*}}\right) u^{*}+\delta^{T^{*}+2} U
$$

where $u^{\prime}=\max _{(e, s)} E u\left(e, s, w_{\theta}^{c}, x_{\theta}^{c}\right)$. Substituting the expressions for $U$ and $U_{1}$ in $\mathrm{IC}_{4}$, we obtain the equivalent form:

$$
0 \geq(1-\delta)\left(u^{\prime}-\widehat{u}\right)+\delta^{T^{*}+1}\left(\widehat{u}-u^{*}\right)-\delta\left(\widehat{u}-u^{*}\right)-\frac{(1-\delta)}{\Delta}\left(1-\delta^{T^{*}+1}\right)\left(u^{c}-\widehat{u}\right),
$$

and it is easy to see that this inequality holds for $T^{*}$ large enough and $\delta$ sufficiently close to 1. For instance, take $T^{*}=+\infty$, then, the inequality is true for all $\delta$ greater than some $\delta_{0}<$ 1 , because $u^{*}<\widehat{u}<u^{c}=E u_{\theta}^{c}$, and $\lim _{\delta \rightarrow 1}(1-\delta) / \Delta<\infty$.

If the state is $\theta=\bar{\theta}$, then the union doesn't deviate from $(\widehat{e}, \widehat{s})$ in the next period if and only if,

$$
U_{\bar{\theta}} \geq(1-\delta) u_{\bar{\theta}}^{c}+\delta(1-\delta) \widehat{u}^{\prime}+\delta^{2}\left(1-\delta^{T^{*}}\right) u^{*}+\delta^{T^{*}+2} U
$$

where $\widehat{u}^{\prime}=\max _{(e, s)} E u(e, s, \widehat{w}, \widehat{x})$. Substituting again the expressions for $U$ and $U_{1}$ in $\mathrm{IC}_{5}$, we obtain a similar result.

Finally, we must check that the union (resp. the employer) prefers to punish the employer (resp. the union) in the case of an off-schedule deviation of the latter, knowing that the employer (resp. the union) punishes the union (resp. the employer) for not punishing by reverting to $\left(w^{*}, x^{*}\right)$ for $T^{*}$ periods. This is true iff

$$
\left(1-\delta^{T^{*}}\right) u^{*}+\delta^{T^{*}} U \geq(1-\delta) \max _{(e, s)} u\left(e, s, w^{*}, x^{*}\right)+\delta\left(1-\delta^{T^{*}}\right) u^{*}+\delta^{T^{*}+1} U
$$

Given that, by definition of the one-shot Nash equilibrium, $u^{*}=\max _{(e, s)} u\left(e, s, w^{*}, x^{*}\right)$, if $T^{*}<\infty$, the above inequality boils down to $u^{*} \leq U$, which is true because $u^{c}>\widehat{u}$ and therefore, $U=\widehat{u}+((1-\delta) / \Delta)\left(u^{c}-\widehat{u}\right)>\widehat{u}>u^{*}$.

We conclude that off-schedule deviations can all be deterred by reversions to the Nash equilibrium of $G$ during $T^{*}$ periods, if players are sufficiently patient and $T^{*}$ is sufficiently large.

Q.E.D. 


\section{Proof of Result 6 .}

To complete the proof of Result 6 , set $\widehat{s}=0$. This implies $\widehat{A}=\mu \widehat{b}$, and it is easy to check that the interior of the intersection of the $\mathrm{IR}_{12}$ and $\mathrm{IC}_{12}$ intervals, if nonempty, is the interval,

$$
b^{\min }=(\widehat{w}-1) \max \left\{\frac{\varphi \mu}{\bar{\theta}+\pi \varphi}, \frac{1}{\pi}\right\}<b<\mu(\widehat{w}-1) \min \left\{\frac{\varphi}{1+\pi \varphi}, \frac{1}{\pi}\right\}=b^{\max } .
$$

We trivially have,

$$
b^{\max }=\frac{\varphi}{1+\pi \varphi}(\widehat{w}-1) \mu .
$$

To prove the latter assertion, Compute first the derivative,

$$
\frac{\partial \varphi}{\partial T}=-\frac{(1-\delta)}{\Delta^{2}} \delta^{T+1} \ln (\delta)>0
$$

as if $T$ was a real number. This shows that $\varphi$ is an increasing function of $T$. In addition, it is easy to check that,

$$
\lim _{T \rightarrow \infty}(1-\pi) \varphi=\frac{(1-\pi) \delta}{1-\delta+(1-\pi) \delta}<1
$$

This shows that $\varphi(1-\pi)<1$. We can now check that $b^{\min }=(\widehat{w}-1) / \pi$ because $\bar{\theta}+\pi \varphi>\varphi \mu \pi$, or equivalently, $\bar{\theta}>\varphi \pi(1-\pi)(\bar{\theta}-1)$, and the latter inequality is true since $\varphi \pi(1-\pi)<1$. Thus, if $\widehat{s}=0$, we have

$$
b^{\min }=\frac{(\widehat{w}-1)}{\pi}, \quad \text { and } \quad b^{\max }=\frac{\varphi \mu(\widehat{w}-1)}{1+\pi \varphi}
$$

The interval is then nonempty if and only if $b^{\min }<b^{\max }$, that is, iff, $1<\pi \varphi(\mu-1)$, or equivalently,

$$
1<\pi(1-\pi) \varphi(\bar{\theta}-1)
$$

This is obviously true if $\bar{\theta}>\bar{\theta}_{0}$. Remark that, using l'Hôpital's rule, we obtain

$$
\lim _{\delta \rightarrow 1} \varphi=\frac{T}{1+T(1-\pi)}, \quad \lim _{\delta \rightarrow 1}\left(\frac{\varphi}{1+\pi \varphi}\right)=\frac{T}{1+T}
$$

and

$$
\lim _{T \rightarrow+\infty} \varphi=\frac{\delta}{1-\delta \pi}, \quad \lim _{T \rightarrow+\infty}\left(\frac{\varphi}{1+\pi \varphi}\right)=\delta .
$$

The ratios $\varphi /(1+\pi \varphi)$ and $\varphi /(\bar{\theta}+\pi \varphi)$ remain bounded when $T$ grows arbitrarily large and when $\delta \rightarrow 1$. Now, if $b$ is chosen in the open interval $\left(b^{\min }, b^{\max }\right)$, by continuity, we can choose a sufficiently small $\widehat{s}>0$ and still satisfy $\mathrm{IC}_{12}$ and $\mathrm{IR}_{12}$. This shows that if $\bar{\theta}>\bar{\theta}_{0}$, 
there exists a pair $(\widehat{s}, \widehat{w})$, with $\widehat{s}>0$ and $\widehat{w}>1$, such that $\mathrm{IC}_{12}$ and $\mathrm{IR}_{12}$ simultaneously hold.

Q.E.D.

\section{Proof of Proposition 2.}

The proof of Proposition 2 is easy if we use $u^{*}$ and $v^{*}$ instead of $\widehat{u}$ and $\widehat{v}$ in the definition of the value functions $U$ and $V$. We can write all incentive constraints in a parallel way, as in the derivation of Results 4-6. It is then easy to check that the honesty-and-courage constraints $\mathrm{IC}_{12}$ can be written,

$$
\frac{\varphi A^{*}}{\bar{\theta}+\pi \varphi} \leq b \leq \frac{\varphi A^{*}}{1+\pi \varphi}
$$

where, $A^{*}=\mu\left[\bar{h}\left(1-x_{1}^{*}\right)+C\left(x_{1}^{*}\right)-1\right]$. It is easy to check that $A^{*}>0$ since $\bar{h}>1$. In addition, we must have $u^{c}>u^{*}$ and $v^{c}>v^{*}$, that is, after some rearrangement of terms,

$$
u^{*}=(1-\underline{e}) \bar{h}\left(1-x_{1}^{*}\right)<\pi b<A^{*}
$$

We then find that the bonus $b$ should be chosen in the open interval $\left(b^{\min }, b^{\max }\right)$, with,

$$
b^{\min }=\max \left\{\frac{u^{*}}{\pi}, \frac{\varphi A^{*}}{\bar{\theta}+\pi \varphi}\right\}, \quad \text { and } \quad b^{\max }=\min \left\{\frac{A^{*}}{\pi}, \frac{\varphi A^{*}}{1+\pi \varphi}\right\} .
$$

If $\underline{e}$ is sufficiently close to 1 , then $b^{\min }=\varphi A^{*} /(\bar{\theta}+\pi \varphi)$. It is easy to see that $b^{\max }=$ $\varphi A^{*} /(1+\pi \varphi)$ since $\bar{\theta}>1$. Thus, $b^{\min }<b^{\max }:$ the interval is nonempty.

Q.E.D.

\section{Proof of Result 7.}

We first prove that $I R$ is binding at the optimum. Suppose that this is false. Then choose small variations $d w_{1}>0, d w_{\bar{\theta}}>0$, such that $d A_{1}-d A_{\bar{\theta}}=0$ : these small variations do not change $I C a b$. The left-hand side of $I R$ decreases by a small amount and the objective increases, a contradiction.

We express $w_{1}$ using $I R$. After some rearrangement of terms, we find,

$$
\pi w_{1} h_{1}\left(1-x_{1}\right)\left(1-s_{1}\right)=v_{0}-\underline{v}-(1-\pi)\left[v_{0} s_{\bar{\theta}}\left(1-x_{\bar{\theta}}\right)+\bar{\theta} A_{\bar{\theta}}\right]-\pi\left[C\left(x_{1}\right)+v_{0} s_{1}\left(1-x_{1}\right)\right]
$$


We then substitute the expression for $w_{1}$ in the objective function. This yields the objective function,

$$
\begin{aligned}
E\left(u_{\theta}\right)= & v_{0}-\underline{v}-(1-\pi)\left[v_{0} s_{\bar{\theta}}\left(1-x_{\bar{\theta}}\right)+\bar{\theta} A_{\bar{\theta}}\right]-\pi\left[C\left(x_{1}\right)+v_{0} s_{1}\left(1-x_{1}\right)\right] \\
& +(1-\pi)\left(w_{\bar{\theta}}-e_{\bar{\theta}}\right) h_{\bar{\theta}}\left(1-x_{\bar{\theta}}\right)\left(1-s_{\bar{\theta}}\right)-\pi e_{1} h_{1}\left(1-x_{1}\right)\left(1-s_{1}\right)
\end{aligned}
$$

We also substitute the expression for $w_{1}$ in $I C b$. This yields,

$$
v_{0}-\underline{v} \leq v_{0} s_{\bar{\theta}}\left(1-x_{\bar{\theta}}\right)+\mu A_{\bar{\theta}}
$$

Since $(I C b b)$ doesn't depend on $\left(e_{1}, s_{1}, x_{1}\right)$, it is optimal to choose $e_{1} h_{1}=\bar{e} \underline{h}=1$. In addition, under Assumption 4, we have,

$$
\frac{\partial E\left(u_{\theta}\right)}{\partial s_{1}}=\pi\left(1-x_{1}\right)\left(1-v_{0}\right) \leq 0
$$

and it is now apparent that $s_{1}=0$ is an optimal choice. Given these results, we find,

$$
\frac{\partial E\left(u_{\theta}\right)}{\partial x_{1}}=-\pi \beta x_{1} \leq 0
$$

and therefore, $x_{1}=0$ is optimal. It is then easy to check that the maximization of $E\left(u_{\theta}\right)$ is equivalent to minimization of the following cost function, denoted $L$.

$$
L=v_{0} s_{\bar{\theta}}\left(1-x_{\bar{\theta}}\right)+(\bar{\theta}-1) w_{\bar{\theta}} h_{\bar{\theta}}\left(1-x_{\bar{\theta}}\right)\left(1-s_{\bar{\theta}}\right)+e_{\bar{\theta}} h_{\bar{\theta}}\left(1-x_{\bar{\theta}}\right)\left(1-s_{\bar{\theta}}\right)+\bar{\theta} C\left(x_{\bar{\theta}}\right),
$$

subject to $(I C b b)$.

Next, we show that $I C b$ must bind at any optimum if $v_{0}$ is large enough. Suppose that this is false. Then, we have $v_{0}-\underline{v}<v_{0} s_{\bar{\theta}}\left(1-x_{\bar{\theta}}\right)+\mu A_{\bar{\theta}}$. Suppose now that $s_{\bar{\theta}}=0$. Then, the constraints $I C a b$ imply $s_{1}\left(1-x_{1}\right)=0$ and $A_{1}-A_{\bar{\theta}}=0$, but this means that $I C b$ is binding, a contradiction. Hence, we must have $s_{\bar{\theta}}>0$. Consider now a feasible small variation $d s_{\bar{\theta}}<0$. By continuity, the right-hand side of $(I C b b)$ varies by a small amount, so that $(I C b b)$ continues to hold if $-d s_{\bar{\theta}}$ is small enough and at the same time, the expected cost varies by the amount

$$
d L=\left(1-x_{\bar{\theta}}\right)\left[v_{0}-(\bar{\theta}-1) w_{\bar{\theta}} h_{\bar{\theta}}-e_{\bar{\theta}} h_{\bar{\theta}}\right] d s_{\bar{\theta}}
$$

If $v_{0}-(\bar{\theta}-1) w_{\bar{\theta}} h_{\bar{\theta}}-e_{\bar{\theta}} h_{\bar{\theta}}>0$, we find that that $d L<0$ and $(I C b b)$ must bind. We assume that $v_{0}$ is large enough so that $v_{0}-(\bar{\theta}-1) w_{\bar{\theta}} h_{\bar{\theta}}-e_{\bar{\theta}} h_{\bar{\theta}}>0$ and will check that this is true 
at the end of our computation. Using $(I C b b)$ expressed as an equality, we eliminate $w_{\bar{\theta}}$ from the objective $L$. Substituting $\mu w_{\bar{\theta}} h_{\bar{\theta}}\left(1-x_{\bar{\theta}}\right)\left(1-s_{\bar{\theta}}\right)=v_{0}-\underline{v}-v_{0} s_{\bar{\theta}}\left(1-x_{\bar{\theta}}\right)-\mu C\left(x_{\bar{\theta}}\right)$ in $L$, after some rearrangement of terms, we see that the objective is in fact to minimize,

$$
\widehat{L}=(1 / \mu)\left[v_{0}(1-\pi(\bar{\theta}-1))-\mu e_{\bar{\theta}} h_{\bar{\theta}}\right]\left(1-x_{\bar{\theta}}\right) s_{\bar{\theta}}+e_{\bar{\theta}} h_{\bar{\theta}}\left(1-x_{\bar{\theta}}\right)+C\left(x_{\bar{\theta}}\right) .
$$

Now, two cases arise. Case 1: If $v_{0}(1-\pi(\bar{\theta}-1))<\mu$, minimization of $\widehat{L}$ implies $s_{\bar{\theta}}=1$. It follows that $x_{\bar{\theta}}$ is fully determined as a solution of $(I C b b)$, expressed as an equality. Given the quadratic specification of $C, x_{\bar{\theta}}$ is a feasible solution of the quadratic equation $0=\underline{v}-\left(v_{0}-\mu\right) x_{\bar{\theta}}+\mu \beta x_{\bar{\theta}}^{2}$. The solutions are real and the smallest solution is smaller than 1 if $v_{0}$ is large enough. We choose the solution that minimizes $L=v_{0}\left(1-x_{\bar{\theta}}\right)+\bar{\theta} C\left(x_{\bar{\theta}}\right)$ subject to $x_{\bar{\theta}}$ being smaller than one and being one of the solutions of the quadratic equation. If $v_{0}$ is large enough, the feasible solution is the smallest root,

$$
\widetilde{x}_{\bar{\theta}}=\frac{v_{0}-\mu-\sqrt{\left(v_{0}-\mu\right)^{2}-4 \mu \beta \underline{v}}}{2 \mu \beta} .
$$

Finally, $\left(w_{\bar{\theta}}, h_{\bar{\theta}}, e_{\bar{\theta}}\right)$ are irrelevant because $s_{\bar{\theta}}=1$; we can choose $w_{\bar{\theta}}=1$ and $e_{\bar{\theta}}=\underline{e}$. From $I R$, we derive, $\underline{v}=\pi\left(v_{0}-w_{1}\right)+(1-\pi)\left[x_{\bar{\theta}} v_{0}-\bar{\theta} C\left(x_{\bar{\theta}}\right)\right]$, that is,

$$
w_{1}=(1 / \pi)\left[v_{0}\left(\pi+\widetilde{x}_{\bar{\theta}}(1-\pi)\right)-(1-\pi) \bar{\theta} C\left(\widetilde{x}_{\bar{\theta}}\right)\right]-\underline{v} .
$$

We find that $w_{1} \geq 1$ if $v_{0}$ is large enough, since $\widetilde{x}_{\bar{\theta}}$ is a decreasing function of $v_{0}$. The condition $v_{0}-(\bar{\theta}-1) w_{\bar{\theta}} h_{\bar{\theta}}-e_{\bar{\theta}} h_{\bar{\theta}}>0$ holds under Assumption 4.

Case 2: If $v_{0}(1-\pi(\bar{\theta}-1))>\mu \underline{e} \bar{h}$, then, given the fact that $e_{\bar{\theta}} h_{\bar{\theta}} \geq 1$, minimization of $\widehat{L}$ implies $s_{\bar{\theta}}=0$. This implies in turn that $e_{\bar{\theta}} h_{\bar{\theta}}=1$ is optimal. We now want to minimize $\left(1-x_{\bar{\theta}}\right)+C\left(x_{\bar{\theta}}\right)=1+\beta x_{\bar{\theta}}^{2}$ with respect to $x_{\bar{\theta}}$. This implies $x_{\bar{\theta}}=0$, and by $(I C b b)$, we derive

$$
w_{\bar{\theta}}=\frac{v_{0}-\underline{v}}{\mu} .
$$

Using $I R$, we finally obtain $\underline{v}=v_{0}-\pi w_{1}-(1-\pi) w_{\bar{\theta}}$. We therefore derive,

$$
w_{1}=\frac{v_{0}-\underline{v}}{\mu} .
$$

These values of the wage are obviously greater than one if $v_{0}$ is large enough. The condition $v_{0}-(\bar{\theta}-1) w_{\bar{\theta}} h_{\bar{\theta}}-e_{\bar{\theta}} h_{\bar{\theta}}>0$ used above is now equivalent to

$$
v_{0}\left[1-\frac{(\bar{\theta}-1)}{\mu}\right]+\frac{(\bar{\theta}-1) \underline{v}}{\mu}>1 .
$$


The latter condition holds in Case 2 if $v_{0}$ is large enough, since $\mu>\bar{\theta}-1$.

Case 3: If $\mu \underline{\underline{h}} \geq v_{0}(1-\pi(\bar{\theta}-1))>\mu$, then, if in addition $e_{\bar{\theta}} h_{\bar{\theta}}=1$, we must also choose $s_{\bar{\theta}}=0=x_{\bar{\theta}}$ to minimize $L$ as in Case 2 above, and the solution is the same as that

of Case 2. If $e_{\bar{\theta}} h_{\bar{\theta}}=\underline{e} \bar{h}$, then we must choose $s_{\bar{\theta}}=1$ to minimize $L$ and the solution is the same as in Case 1 above.

Q.E.D.

\section{Proof of Result 8 .}

We check the inequality $R=U+V \geq \widetilde{R}=\widetilde{u}+\widetilde{v}$. Assume that $\widehat{s}=0$, using the expressions of $U$ and $V$ given above, the inequality is equivalent to,

$$
\widetilde{R} \leq v_{0}-(\mu-1) \widehat{w}-1-\gamma(\mu+1)(\widehat{w}+1)
$$

where

$$
\gamma=\frac{1-\delta}{\Delta}, \quad \text { and } \quad R=\widehat{R}+\gamma\left(R^{c}-\widehat{R}\right)
$$

Considering Case 1 in Result $7, \widetilde{R}=\pi\left(v_{0}-1\right)-(1-\pi)\left[v_{0} \widetilde{x}_{\bar{\theta}}+\bar{\theta} C\left(\widetilde{x}_{\bar{\theta}}\right)\right]$, and we must have,

$$
\widehat{w} \leq \frac{(1-\pi)}{(\mu-1)(\gamma+1)}\left[v_{0}-1+v_{0} \widetilde{x}_{\bar{\theta}}+\bar{\theta} C\left(\widetilde{x}_{\bar{\theta}}\right)\right]-\frac{\gamma}{(\gamma+1)}
$$

The term $\widetilde{x}_{\bar{\theta}}$ is defined in the proof of Result 7 . This inequality will always be satisfied if $v_{0}$ is large enough, because in Case 1 , the term $\left(v_{0} \widetilde{x}_{\bar{\theta}}+\bar{\theta} C\left(\widetilde{x}_{\bar{\theta}}\right)\right) \rightarrow \underline{v}$ as $v_{0}$ grows without bound (this is because $\widetilde{x}_{\bar{\theta}} \rightarrow 0$ as $v_{0} \rightarrow+\infty$ ). Hence, in Case 1 , if $v_{0}$ is large enough, there is an open interval of values of $\widehat{w}$ for which $R>\widetilde{R}$, while $R$ is an equilibrium value of surplus, because $\widehat{w}$ is chosen to satisfy $\mathrm{IR}_{12}$ and $\mathrm{IC}_{12}$.

Considering now Case 2 in Result 7 , we have $\widetilde{R}=-1+\underline{v}+\left(v_{0}-\underline{v}\right) / \mu$ and we find that $\widehat{w}$ must satisfy,

$$
\widehat{w} \leq \frac{1}{\gamma+1}\left(\frac{v_{0}-\underline{v}}{\mu}-\gamma\right)
$$

The upper bound is again an increasing function of $v_{0}$. Reasoning as in Case 1 above, we find the same conclusion.

Q.E.D. 


\section{Proof of Result 9.}

Most of the results in the statement of Result 9 are obtained by straightforward calculus. If we now treat $T$ as a real number, we find

$$
\frac{\partial}{\partial T}\left(\frac{1-\delta}{\Delta}\right)=\frac{(1-\delta)}{\Delta^{2}}(1-\pi) \delta^{T+1} \ln (\delta)<0 .
$$

It follows from this that $\partial R / \partial T<0$ and the optimal value of $T$ is therefore the smallest value compatible with $\mathrm{IC}_{12}$ and $\mathrm{IR}_{12}$. Now, going back to $\mathrm{IC}_{12}$, we find that,

$$
\frac{\partial \varphi}{\partial T}=-\frac{(1-\delta)}{\Delta^{2}} \delta^{T+1} \ln (\delta)>0 .
$$

Easy algebra shows that the equation $\varphi \eta=b$ can be rewritten in the form $\delta^{T}=H$, where

$$
H=\frac{\delta \eta-b(1-\delta \pi)}{\delta(\eta-b(1-\pi))}
$$

It follows that the real solution of $\varphi(\delta, \pi, \widetilde{T}) \eta=b$, denoted $\widetilde{T}$, is well-defined if $0<H<1$. It is easy to check that $H<1$ if $\delta<1$. Given this, we get $H>0$ iff $\delta \eta-b(1-\delta \pi)>0$, or equivalently, iff

$$
\frac{\delta}{1-\delta \pi} \eta=\left(\lim _{T \rightarrow \infty} \varphi\right) \eta>b
$$

But the latter strict inequality is true since $\eta=v^{c}-\widehat{v}>0, \varphi$ is a increasing function of $T$ and $\mathrm{IC}_{1}$ holds by definition. We therefore easily find the well-defined real solution,

$$
\widetilde{T}=\frac{\ln (H)}{\ln (\delta)} .
$$

Given that $\varphi \eta=b$ and $\varphi$ is increasing in $T$, we see that $\widetilde{T}$ must be decreasing in $\eta$, and calculus confirms that

$$
\frac{\partial \widetilde{T}}{\partial \eta}=\frac{(1-\delta) b}{\ln (\delta) \delta H[\eta-b(1-\pi)]^{2}}<0 .
$$

After some computations, we obtain the following result:

$$
\frac{\partial \widetilde{T}}{\partial b}=\frac{\partial \widetilde{T}}{\partial w_{1}^{c}}=\frac{-(1-\delta)(\eta+b \pi)}{\ln (\delta)[\delta \eta-b(1-\delta \pi)][\eta-b(1-\pi)]}>0 .
$$

Easy calculus yields,

$$
\frac{\partial \widetilde{T}}{\partial \widehat{s}}=\left(v_{0}-\mu \widehat{w}\right) \frac{\partial \widetilde{T}}{\partial \eta}<0
$$


The minimal admissible value of $\eta$ is $b(1-\delta \pi) / \delta$. When $\eta$ approaches this minimal value, $\widetilde{T} \rightarrow+\infty$. Hence, when $\widehat{s}$ become so small that $\eta$ approaches its minimal value, then $\widetilde{T}$ tends toward infinity.

Finally, it can be checked that $\widetilde{T}$ is a decreasing function of $\delta$ and that $\widetilde{T}$ approaches a finite limit as $\delta \rightarrow 1$, namely,

$$
\frac{\partial \widetilde{T}}{\partial \delta}<0, \quad \text { and } \quad \lim _{\delta \rightarrow 1} \widetilde{T}=\frac{b}{\eta-b(1-\pi)} .
$$

Q.E.D.

\subsection{Is there an optimal probability of strikes?}

We now ask the following question. Given $\widehat{w}, b$ and $\delta$, can we find an optimal combination $(T, \widehat{s})$ ? Note that $\widetilde{T}$ is a function of $\widehat{s}$ and the equilibrium surplus $R$ is a function of $(T, \widehat{s})$. Define $\widetilde{R}(\widehat{s})=R(\widetilde{T}(\widehat{s}), \widehat{s})$. Then, there exists an interior optimum $\widehat{s}$ only if, applying the chain rule, we have,

$$
\frac{d \widetilde{R}}{d \widehat{s}}=\frac{\partial R}{\partial T} \frac{\partial \widetilde{T}}{\partial \widehat{s}}+\frac{\partial R}{\partial \widehat{s}}=0
$$

for a feasible value of $\widehat{s}$. Some simple (but lengthy) computations yield the following result.

Result 10. Given an equilibrium of the form described in Proposition 1 above and given admissible values of $\widehat{w}, w_{1}^{c}$ and $\delta$, the duration of strikes $\widehat{s}$ satisfies the necessary condition $d \widetilde{R} / d \widehat{s}=0$ only if the following proportionality condition holds:

$$
\frac{v^{c}}{u^{c}+v^{c}}=\frac{\widehat{v}}{\widehat{u}+\widehat{v}}=\frac{v_{0}-\mu \widehat{w}}{v_{0}-(\mu-1) \widehat{w}-1} .
$$

In other words, $(T, \widehat{s})$ maximizes $R$ only if the latter proportionality condition holds and $T=\widetilde{T}(\widehat{s})$.

\section{Proof of Result 10.}

Using the notation $\widehat{R}=\widehat{u}+\widehat{v}$, and $R^{c}=u^{c}+v^{c}$, we have,

$$
\frac{\partial R}{\partial T} \frac{\partial \widetilde{T}}{\partial \widehat{s}}+\frac{\partial R}{\partial \widehat{s}}=\frac{\partial \Delta^{-1}}{\partial T}(1-\delta)\left(R^{c}-\widehat{R}\right) \frac{\partial \widetilde{T}}{\partial \widehat{s}}+\left(1-(1-\delta) \Delta^{-1}\right) \frac{\partial \widehat{R}}{\partial \widehat{s}} .
$$


Using the facts that $H=\delta^{\widetilde{T}}$ and $\varphi \eta=b$, where $H$ is defined in the proof of Result 9 , we can simplify the above expression and we obtain,

$$
\frac{\delta(1-\delta)(1-\pi)\left(R^{c}-\widehat{R}\right)}{\Delta^{2}} \frac{b(1-\delta)^{2}\left(v_{0}-\mu \widehat{w}\right)}{(\eta-b(1-\pi))^{2}}=\frac{\delta\left(1-\delta^{\widetilde{T}}\right)(1-\pi)}{\Delta}\left(v_{0}-(\mu-1) \widehat{w}-1\right) .
$$

Using the fact that $\left(1-\delta^{\widetilde{T}}\right)(\eta-b(1-\pi))=b(1-\delta) / \delta$, the above expression can be simplified further and rewritten as follows:

$$
\frac{(1-\delta)\left(R^{c}-\widehat{R}\right)}{\Delta(\eta-b(1-\pi))}=\frac{v_{0}-(\mu-1) \widehat{w}-1}{v_{0}-\mu \widehat{w}} .
$$

Now, recalling that $\Delta=1-\delta \pi-(1-\pi) \delta H$, it is easy to check that $(\eta-b(1-\pi)) \Delta=\eta(1-\delta)$. Thus, we obtain,

$$
\frac{\left(R^{c}-\widehat{R}\right)}{\eta}=\frac{v_{0}-(\mu-1) \widehat{w}-1}{v_{0}-\mu \widehat{w}} .
$$

Using now the fact that $\eta=v^{c}-\widehat{v}$ and multipliying the numerator and denominator of the right-hand side by $(1-\widehat{s})$, we obtain

$$
\frac{R^{c}-\widehat{R}}{v^{c}-\widehat{v}}=\frac{\widehat{R}}{\widehat{v}}
$$

A simple rearrangement yields the result,

$$
\frac{R^{c}}{\widehat{R}}=\frac{v^{c}}{\widehat{v}}
$$

Q.E.D.

It is remarkable that the ratios $\widehat{v} /(\widehat{u}+\widehat{v})$ and $v^{c} /\left(u^{c}+v^{c}\right)$ do not depend on $\widehat{s}$. Therefore, given feasible values of $\widehat{w}$ and $b$, the pair $(T, \widehat{s})$ maximizes $R$, provided that $\widehat{w}$ satisfies the above proportionality condition and $T=\widetilde{T}(\widehat{s})$.

The ratio $\widehat{v} /(\widehat{u}+\widehat{v})$ is a function of $\widehat{w}$. We want to choose $\widehat{w}$ so as to satisfy the proportionality condition of Result 10 above. Let the ratio $\rho=v^{c} /\left(u^{c}+v^{c}\right)$ be fixed. We easily find the appropriate value,

$$
\widehat{w}=\frac{(1-\rho) v_{0}+\rho}{(1-\rho) \mu+\rho} .
$$

And this latter value of $\widehat{w}$ is clearly greater than 1 if $v_{0}$ is large enough. 


\section{References}

Abreu, Dilip, David G. Pearce and Ennio Stachetti (1990), "Toward a Theory of Discounted Repeated Games with Imperfect Monitoring", Econometrica, 58, 1014-1063.

Albert Shanker Institute (2008), A Cry for Justice: The Voices of Chinese Workers, The Albert Shanker Institute, Washington DC.

Ausubel, Lawrence, Cramton, Peter and Raymond Deneckere (2001), "Bargaining with Incomplete Information," Chapter 50 in Robert J. Aumann and Sergiu Hart eds., Handbook of Game Theory, Volume 3, Elsevier, Amsterdam.

Athey, Susan and Kyle Bagwell (2001), "Optimal Collusion with Private Information", Rand Journal of Economics, 32, 428-465.

Baker, George, Gibbons, Robert and Kevin J. Murphy (1994), "Subjective Performance Measures in Optimal Incentive Contracts", Quarterly Journal of Economics, 109, 11251156.

Bull, Clive (1987), "The Existence of Self-Enforcing Implicit Contracts," Quarterly Journal of Economics, 102, 147-159..

Card, David (1990a), "Strikes and Bargaining: A Survey of the Recent Empirical Literature" American Economic Review, AEA Papers and Proceedings, 80, 410-415.

Card, David (1990b), "Strikes and Wages: A Test of an Asymmetric Information Model," Quarterly Journal of Economics, 105, 625-659.

DARES (2009), http://www.travail-solidarite.gouv.fr/etudes-recherche-statistiques-dares; Ministère du Travail, France.

Cramton, Peter and Joseph S. Tracy (1992), "Strikes and Holdouts in Wage Bargaining: Theory and Data," American Economic Review, 82, 100-121.

Devereux, Paul J. and Robert A. Hart (2008), "Good Time to Stay Out? Strikes and the Business Cycles", IZA discussion paper No. 3614, IZA, Bonn, Germany. 
The Economist (2010), "The Next China. As the Supply of Migrant Labour Dwindles, the Workshop of the World is Embarking on a Migration of its Own", July 29th, 2010.

The Economist (2011), "Government Workers of the World Unite!", January 8th, 2011.

Fuchs, William (2007), "Contracting with Repeated Moral Hazard and Private Evaluations", American Economic Review, 97, 1432-1448.

Fudenberg, Drew, Levine, David and Eric Maskin (1994), "The Folk Theorem with Imperfect Public Information," Econometrica, 62, 87-100.

Gary-Bobo, Robert J. and Touria Jaaidane (2006), "Strikes as the 'Tip of the Iceberg' in a Theory of Firm-Union Cooperation", CEPR discussion paper no 6644, CEPR, London.

Green, Edward J., and Robert M. Porter (1984), "Noncooperative Collusion under Imperfect Price Information"," Econometrica, 52, 997-1039.

Hale, Dominic (2008), "International Comparisons of Labour Disputes", Office of National Statistics, Economic and Labour Market Review, 2, 32-39.

Hansen, Alvin (1921), "Cycles of Strikes", American Economic Review, 11, 616-621.

Harrison, Alan, and Mark Stewart (1994), "Is Strike Behavior Cyclical?", Journal of Labor Economics, 12, 524-553.

Hayes, Beth (1984), "Unions and Strikes with Asymmetric Information", Journal of Labor Economics, 2, 57-83.

Jaaidane, Touria and Robert J. Gary-Bobo (2008), "Salaires et carrières dans la fonction publique: le cas des éboueurs", Revue Française d'Economie, 22, 3-59.

Kennan, John (1986), "The Economics of Strikes", in Orley Ashenfelter and Richard Layard eds., Handbook of Labor Economics, North-Holland, Amsterdam.

Kennan John and Robert Wilson (1989), "Strategic Bargaining Models and Interpretation of Strike Data," Journal of Applied Econometrics, 4, supplement, S87-S130. 
Kennan John and Robert Wilson (1993), "Bargaining with Private Information," Journal of Economic Literature, 31, 45-104.

Krueger, Alan, B. and Alexandre Mas (2004), "Strikes, Scabs and Tread Separations: Labor Strife and the Production of Defective Bridgestone/Firestone Tires", Journal of Political Economy, 112, 253-289.

Laffont Jean-Jacques and David Martimort (2001), The Theory of Incentives: The PrincipalAgent Model, Princeton University Press, Princeton, New Jersey.

Levin, Jonathan (2003) "Relational Incentive Contracts", American Economic Review, 93, $835-857$.

Mailath, George J. and Larry Samuelson (2006), Repeated Games and Reputations. LongRun Relationships, Oxford University Press, Oxford, UK.

Malcomson, James M. and Franz Spinnewyn (1988), "The Multi-Period Principal-Agent Problem," Review of Economic Studies, 55, 391-407.

MacLeod, W. Bentley and James M. Malcomson, (1989), "Implicit Contracts, Incentive Compatibility, and Involuntary Unemployment," Econometrica, 57, 447-480.

MacLeod, W. Bentley and James M. Malcomson, (1998), "Motivation and Markets," American Economic Review, 88, 388-411.

MacLeod, W. Bentley (2003), "Optimal Contracting with Subjective Evaluation," American Economic Review, 92, 216-240.

McConnell, Sheena (1990), "Cyclical Fluctuations in Strike Activity", Industrial and Labor Relations Review, 44, 130-143.

Mas, Alexandre (2006), "Pay, Reference Points and Police Performance", Quarterly Journal of Economics, 121, 783-821.

Rees, Albert (1952), "Industrial Conflict and Business Fluctuations", Journal of Political Economy, 60, 371-382. 
Robinson, James A. (1999), "Dynamic Contractual Enforcement: A Model of Strikes," International Economic Review, 40, 209-229.

Vroman, Susan, B. (1989) "A Longitudinal Analysis of Strike Activity in U.S. Manufacturing: 1957-1984", American Economic Review, 79, 816-826.

Zhao, Rui R. (2009), "Productive Low Morale", Economics Letters, 103, 18-22. 
Fig. 1. Number of working days lost due to strikes

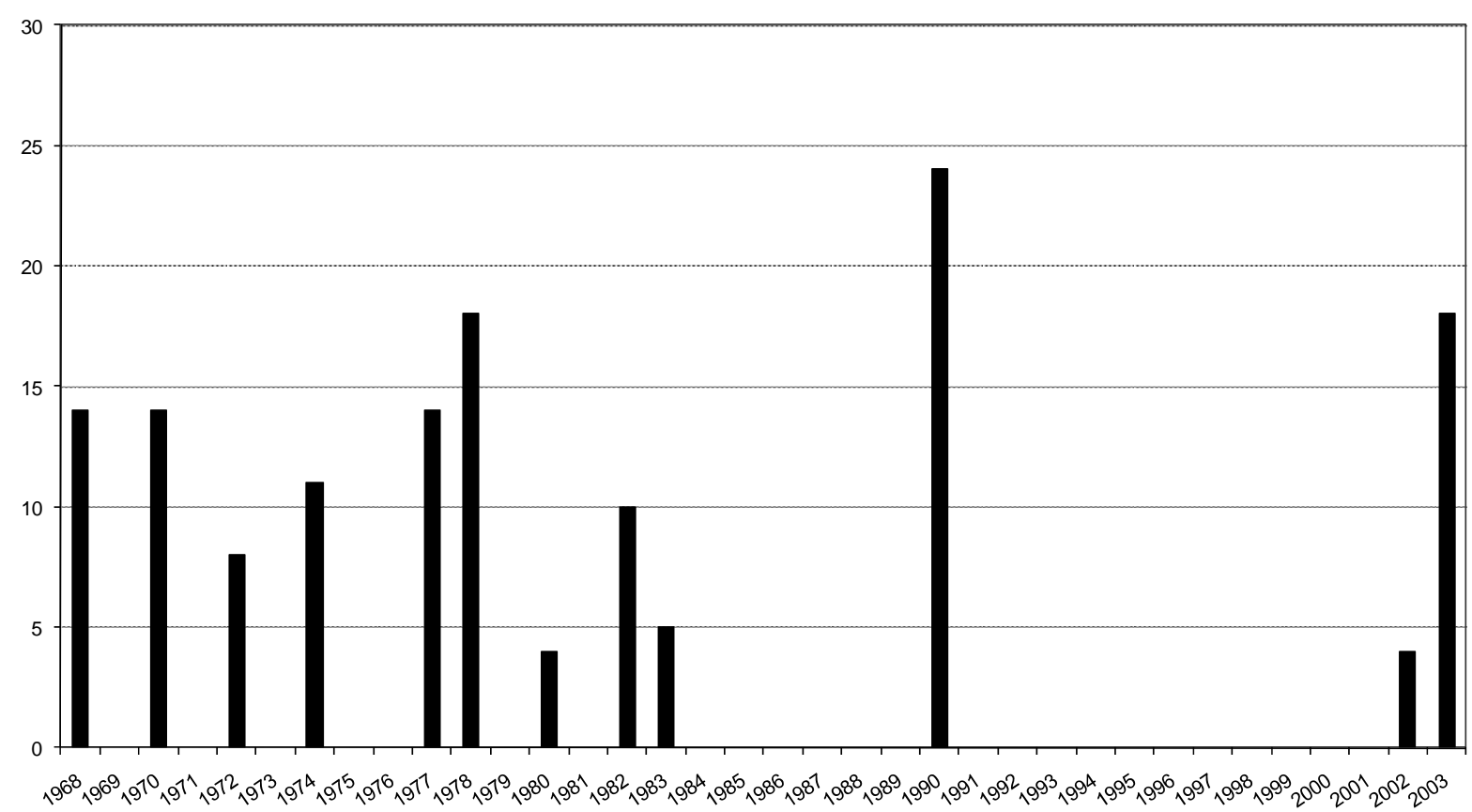

Fig 2. Change in the number of dustmen with civil servant status

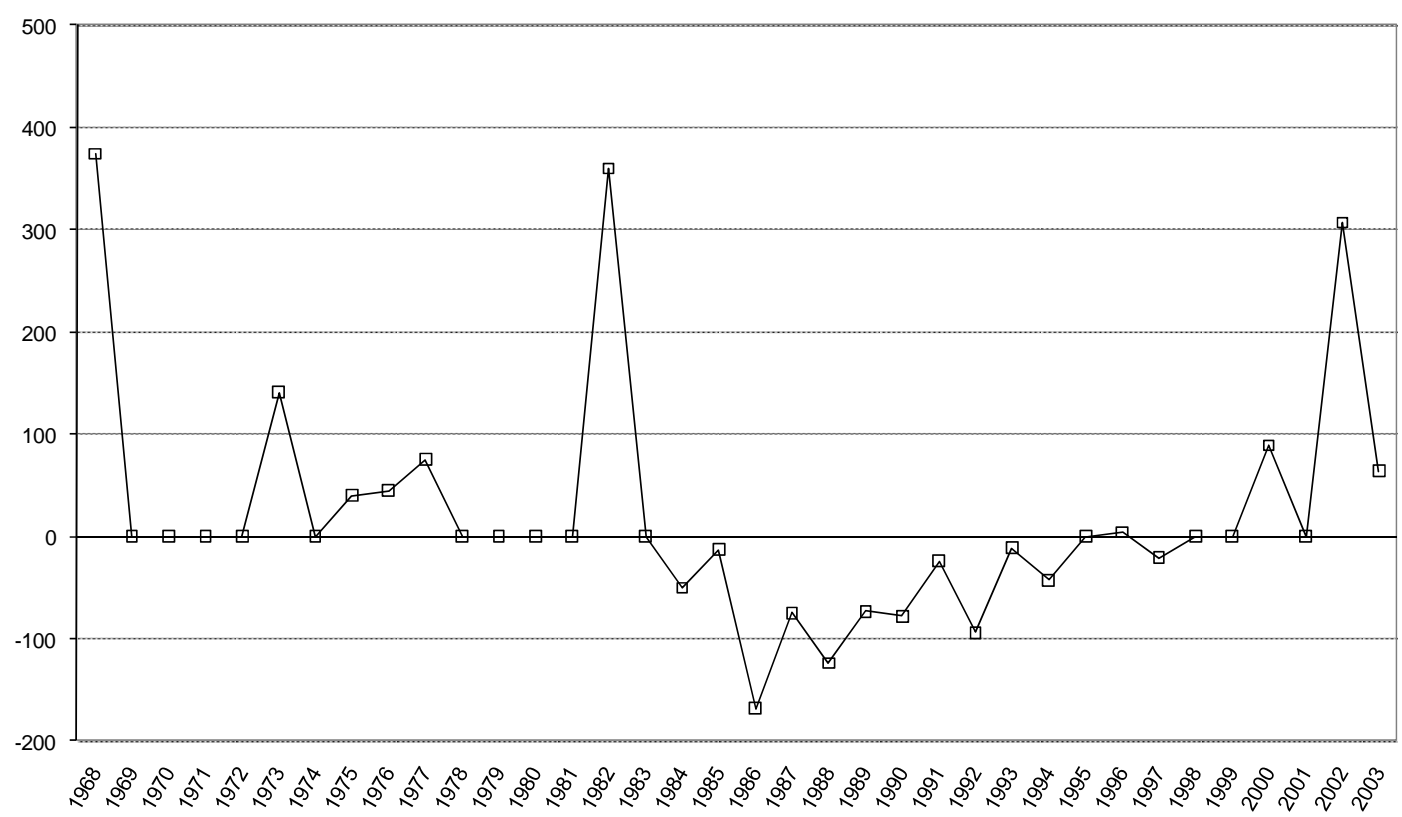




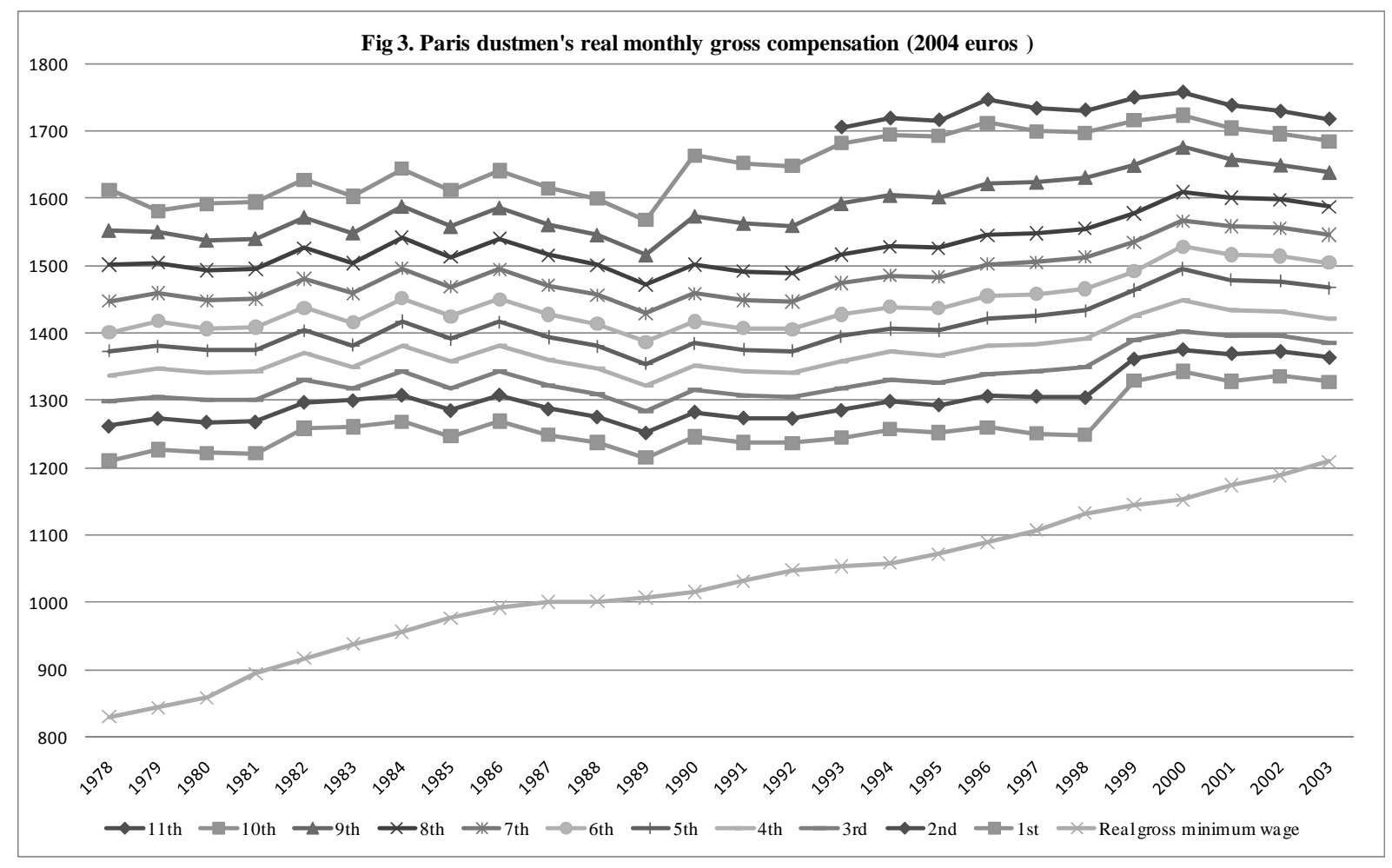


Fig 4. Simulation of a Paris dustman's career:

impact of the seniority rules on real monthly take-home pay

1600

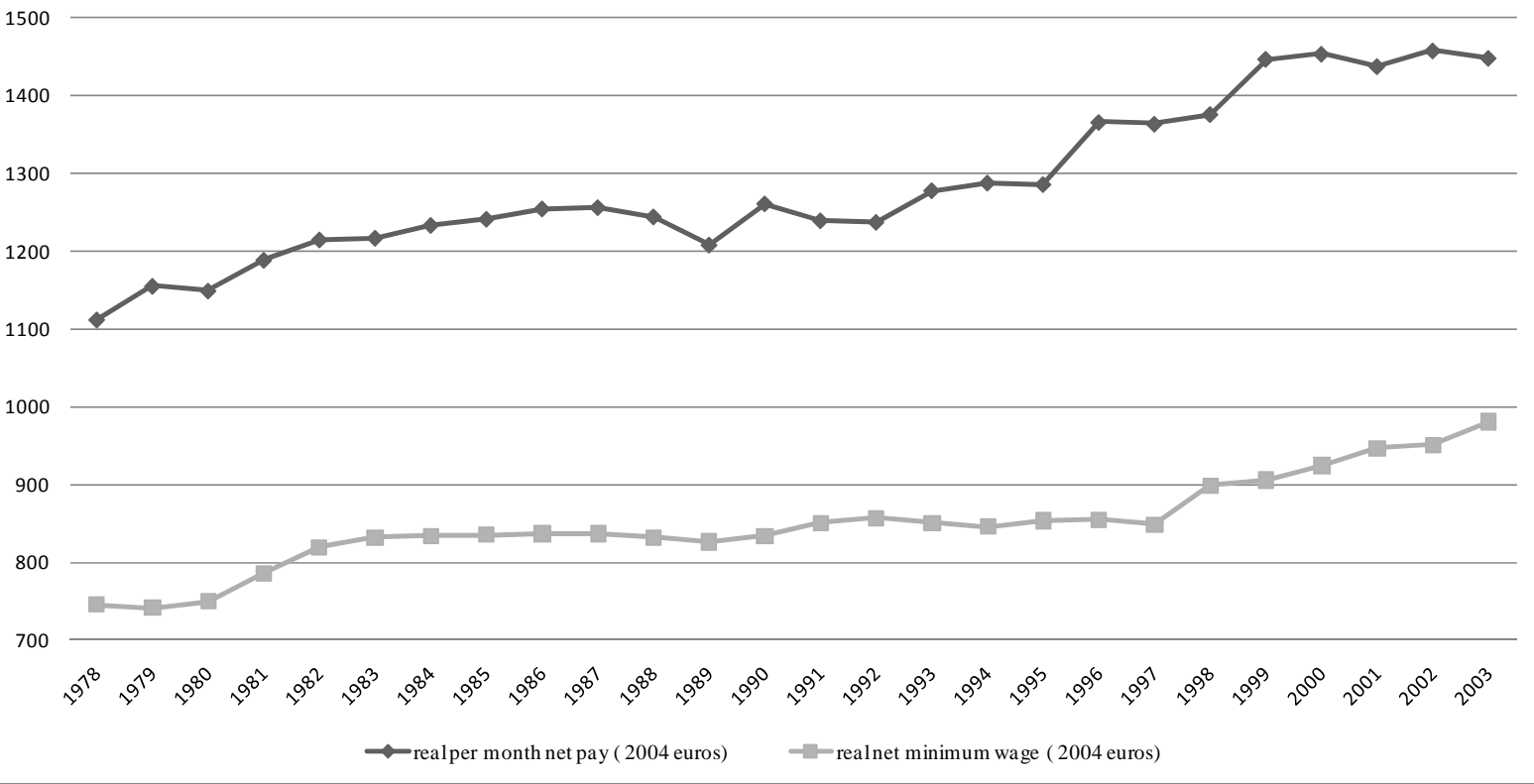

Fig. 5. Evolution of real career value of a Paris dustman with myopic expectations and a $3 \%$ discount rate

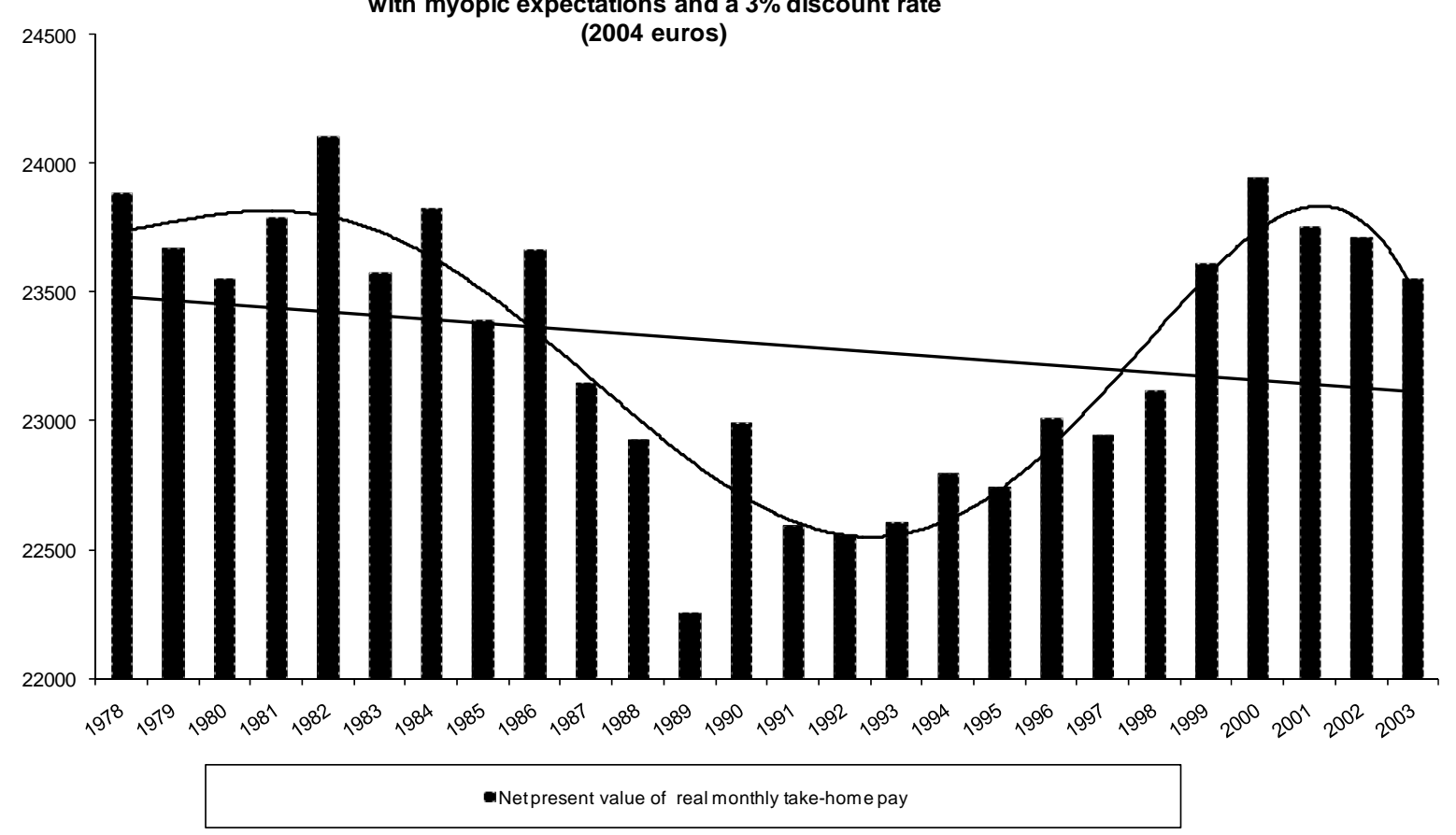




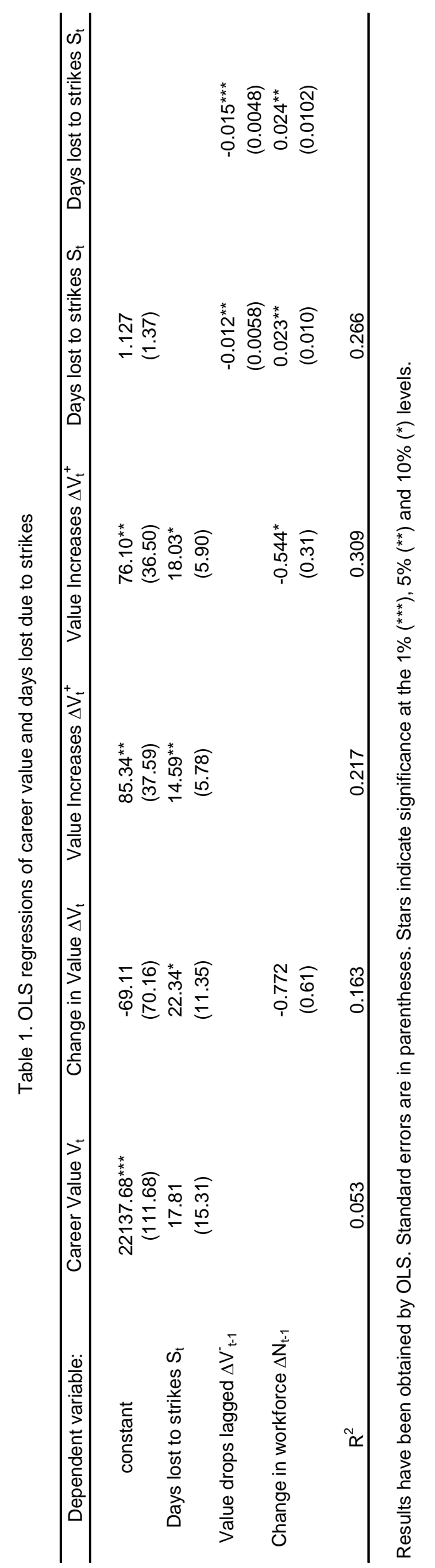

